\title{
All-polymeric transient neural probe for prolonged in-vivo electrophysiological recordings
}

\author{
Laura Ferlauto $^{\mathrm{a}, 1,2}$, Paola Vagni ${ }^{\mathrm{a}, 1,3}$, Adele Fanelli ${ }^{\mathrm{a}}$, Elodie Geneviève Zollinger ${ }^{\mathrm{a}}$, \\ Katia Monsorno $^{\mathrm{b}}$, Rosa Chiara Paolicelli ${ }^{\mathrm{b}}$, Diego Ghezzi ${ }^{\mathrm{a} \text {, * }}$ \\ ${ }^{a}$ Medtronic Chair in Neuroengineering, Center for Neuroprosthetics and Institute of Bioengineering, School of Engineering, École polytechnique fédérale de Lausanne, \\ Switzerland \\ ${ }^{\mathrm{b}}$ Department of Biomedical Sciences, Faculty of Biology and Medicine, University of Lausanne, Switzerland
}

\section{A B S T R A C T}

Transient bioelectronics has grown fast, opening possibilities never thought before. In medicine, transient implantable devices are interesting because they could eliminate the risks related to surgical retrieval and reduce the chronic foreign body reaction. Despite recent progress in this area, the potential of transient bioelectronics is still limited by their short functional lifetime owed to the fast dissolution rate of degradable metals, which is typically a few days or weeks. Here we report that a switch from degradable metals to an entirely polymer-based approach allows for a slower degradation process and a longer lifetime of the transient probe, thus opening new possibilities for transient medical devices. As a proof-of-concept, we fabricated all-polymeric transient neural probes that can monitor brain activity in mice for a few months, rather than a few days or weeks. Also, we extensively evaluated the foreign body reaction around the implant during the probe degradation. This kind of devices might pave the way for several applications in neuroprosthetics.

\section{Introduction}

Building devices able to disappear in the surrounding environment after a programmed lifetime, leaving minimal and harmless traces after their disposal, is a fascinating idea for the development of green electronics to preserve the environment by reducing waste production and recycling processes [1-5]. The same concept is also appealing for medical devices [6-8], such as localised drug release systems [9], power units [10], external sensors [11] and implantable recording systems [12], to eliminate the risks related to surgical retrieval [13] and reduce the chronic foreign body reaction [14].

In medical applications, the durability of transient devices is highly dependent on the degradation time and process of the materials used for the substrate and encapsulation layers and, most importantly, of the functional components exposed to the body. While several biodegradable natural or synthetic polymers, such as cellulose [1], silk [15] and poly(lactic-co-glycolic acid) [16,17], are often used as substrate and encapsulation materials, so far the choice for the functional elements has always landed on transient inorganic materials. These materials, such as magnesium, zinc, molybdenum, or iron, dissolve within minutes, hours, or maximum days, once in contact with body fluids [11,18]. This short functional window still limits the list of potential medical applications for transient devices.

To extend the lifetime of transient medical devices, and consequently increase their possible applications, such as for example mid-term monitoring of the brain activity, our strategy is to switch from degradable metals to entirely polymer-based devices. The results show the fabrication and the in-vitro characterization of all-polymeric transient neural probes (TNPs) allowing prolonged electrophysiological in-vivo recordings up to three months post-implantation. Histological studies revealed minimal foreign body reaction both in the short- and long-term.

\section{Materials and methods}

\subsection{Fabrication of transient all-polymeric neural probe}

Two 4-inch silicon wafers (525- $\mu \mathrm{m}$ thick) were etched (AMS 200, Alcatel) to obtain the positive mould for the substrate layer and the

\footnotetext{
* Corresponding author.

E-mail address: diego.ghezzi@epfl.ch (D. Ghezzi).

1 These authors contributed equally to this work.

${ }^{2}$ Present address: Department of Physics and Interdepartmental Centre of Industrial Research in Advanced Mechanical Engineering Applications and Materials Technology, University of Bologna, Italy.

${ }^{3}$ Present address: Department of Developmental Genetics, Skirball Institute of Biomolecular Medicine, New York University, USA.
} 
negative mould for the encapsulation layer (both structures are $100-\mu \mathrm{m}$ thick). Polycaprolactone (PCL, MW 50,000; 25,090, Polyscience) in powder was dissolved in chloroform (C2432, Sigma-Aldrich) at a concentration of $0.25 \mathrm{~g} \mathrm{ml}^{-1}$ and was left stirring and heating at $60{ }^{\circ} \mathrm{C}$ overnight over a hotplate. The day after, $6 \mathrm{ml}$ of PCL solution were spincoated on both silicon moulds (positive: $150 \mathrm{rpm}$ for $30 \mathrm{~s}$; negative: 200 rpm for $30 \mathrm{~s}$ ) pre-treated with chlorotrimethylsilane (92,361, SigmaAldrich) to prevent permanent attachment of the PCL layers. The wafers were then left $30 \mathrm{~min}$ in the oven at $75{ }^{\circ} \mathrm{C}$ to let the chloroform evaporate. After a cool-down period of at least $2 \mathrm{~h}$, the PCL layer from the positive wafer was peeled off, and the design (electrodes, traces and pads) was filled with an aqueous solution of PEDOT:PSS:EG, which is poly(3,4-ethylenedioxythiophene)-poly(styrenesulfonate) (PEDOT:PSS; M122 PH1000, Ossila) mixed with 20 wt\% ethylene glycol (EG; 324,558 , Sigma-Aldrich), and cured overnight in an oven at $37^{\circ} \mathrm{C}$. The PCL layer from the negative wafer, instead, was peeled off after lasercutting the openings for the electrodes and pads (Optec MM200-USP). Eventually, the two layers were manually aligned and fused together over a hotplate at $60{ }^{\circ} \mathrm{C}$ for a few seconds. A silver-based epoxy (H27D Kit Part A, Epo-Tek) was then applied on the pads and connectors were positioned and immobilised with silicone (DC 734 RTV clear, Dow Corning). Three types of all-polymeric TNPs were fabricated with a different number of electrodes: 4 electrodes $(500-\mu \mathrm{m}$ in diameter; connector 143-56-801, Distrelec), 1 electrode (500- $\mu \mathrm{m}$ in diameter; no connector) and 17 electrodes $(700-\mu \mathrm{m}$ in diameter; connector 61001821821, Wurth Elektronik). Samples with fluorescent PEDOT: PSS:EG were prepared as previously described [19]. Briefly, poly(vinyl alcohol) (PVA, MW 130,000; 563,900, Sigma-Aldrich) was dissolved in deionization water $5 \mathrm{wt} \%$. A composite solution (25 wt\%) of PVA and PEDOT:PSS:EG was prepared, deposited on the PCL, and baked $\left(50{ }^{\circ} \mathrm{C}\right.$, 10 min). (3-Glycidyloxypropyl)trimethoxylane (440,167, Sigma-Aldrich) was then deposited by vapour deposition at $90{ }^{\circ} \mathrm{C}$ for 45 min. Afterwards, a solution of fluorescein isothiocyanate (FITC) labelled poly-L-lysine (P3543, Sigma-Aldrich) in phosphate buffered saline (PBS; $50 \mu \mathrm{g} \mathrm{ml}^{-1}$ ) was drop-casted on the sample and allowed to react for $2 \mathrm{~h}$ at room temperature. The sample was then rinsed with PBS $(0.1 \mathrm{M})$, sodium chloride $(0.1 \mathrm{M})$, and deionised water.

\subsection{Scanning electron microscopy}

Images were taken with a Schottky field emission scanning electrons microscope (SU5000, Hitachi) and image post-processing was performed with ImageJ.

\subsection{Electrochemistry}

Electrochemical characterization was performed with a threeelectrode potentiostat (Compact Stat, Ivium) at room temperature. Each all-polymeric TNP was immersed in PBS (pH 7.4) together with a silver/silver chloride reference wire and a platinum counter wire. Impedance spectroscopy (IS) was measured between $1 \mathrm{~Hz}$ and $1 \mathrm{MHz}$ using an AC voltage of $50 \mathrm{mV}$.

\subsection{Resistance measures}

Line resistance was measured using a data acquisition and logging digital multimeter system (daq6510, Keithley).

\subsection{Cytotoxicity test}

A test on extract was performed on two all-polymeric TNPs (4-electrodes design) sterilised by UV exposure, with a ratio of the product to extraction vehicle of $3 \mathrm{~cm}^{2} \mathrm{ml}^{-1}$. The extraction vehicle was Eagle's minimum essential medium (11090081, Thermo Fisher Scientific) supplemented with $10 \%$ foetal bovine serum (10270106, Thermo Fisher Scientific), $1 \%$ penicillin-streptomycin (15070063, Thermo Fisher
Scientific), $2 \mathrm{mM} \mathrm{L-Glutamine} \mathrm{(25030081,} \mathrm{Thermo} \mathrm{Fisher} \mathrm{Scientific)}$ and $2.50 \mu \mathrm{g} \mathrm{ml}^{-1}$ Amphotericin B (15290026, Thermo Fisher Scientific). The extraction was performed for $24 \mathrm{~h}$ at $37{ }^{\circ} \mathrm{C}$ and $5 \% \mathrm{CO}_{2}$. L929 cells (88102702, Sigma-Aldrich) were plated in a 96-well plate at a subconfluent density of 7000 cells per well in $100 \mu \mathrm{l}$ of the same medium. L929 cells were incubated for $24 \mathrm{~h}$ at $37{ }^{\circ} \mathrm{C}$ and $5 \% \mathrm{CO}_{2}$. After incubation, the medium was removed from the cells and replaced with the extract ( $100 \mu \mathrm{l}$ per well). After another incubation of $24 \mathrm{~h}, 50 \mu \mathrm{l}$ per well of XTT reagent (Cell proliferation kit 11465015001, Sigma-Aldrich) were added and incubated for $4 \mathrm{~h}$ at $37{ }^{\circ} \mathrm{C}$ and $5 \% \mathrm{CO}_{2}$. An aliquot of $100 \mu \mathrm{l}$ was then transferred from each well into the corresponding wells of a new plate, and the optical density was measured at $450 \mathrm{~nm}$ by using a plate reader (FlexStation3, MolecularDevices). Clean medium alone was used as a negative control, whereas medium supplemented with $15 \%$ of dimethyl sulfoxide (D2650-5X5ML, Sigma-Aldrich) was used as a positive control.

\subsection{Accelerated degradation}

All-polymeric TNPs were prepared as described above, with the 4electrode design. After fabrication, samples were weighted to set the initial weight value before starting degradation. Degradation of each sample occurred in $10-\mathrm{ml} \mathrm{PBS}$ at $37^{\circ} \mathrm{C}$ and was accelerated by $\mathrm{pH}$ increase: $100 \mu \mathrm{l}$ of $\mathrm{NaOH}$ ( $2 \mathrm{M} \mathrm{NaOH}$ Standard solution, 71,474-1L, Fluka) were added to the solution to reach $\mathrm{pH} 12$. At each time point, samples were first gently dried with a tissue and then let dry completely under vacuum at room temperature for $4 \mathrm{~h}$. Once dry, samples were weighted. Normalised weight for each sample was computed as the ratio between the weight at each time point and the initial value.

\subsection{Animal handling}

All experiments were conducted according to the animal authorizations GE13416 approved by the Département de l'emploi, des affaires sociales et de la santé (DEAS), Direction générale de la santé of the Republique et Canton de Geneve in Switzerland and VD3420 approved by Service de la consommation et des Affaires vétérinaires (SCAV) of the Canton de Vaud in Switzerland. All the experiments were carried out during the day cycle. For the entire duration of the experiment, the health condition was evaluated three times a week, and the bodyweight was controlled once a week. Experiments were performed in adult $(>1$ month-old) mice. Male and female C57BL/6J mice (Charles River) were kept in a $12 \mathrm{~h}$ day/night cycle with access to food and water ad libitum. White light (300 \pm 50 lux) was present from 7 a.m. to 7 p.m. and red light (650-720 nm, 80-100 lux) from 7 p.m. to 7 a.m. Homozygous B6.129P2(Cg)-Cx3 $\mathrm{cr}^{\mathrm{tm} 2.1 \text { (cre/ERT2)Litt } / W g a n J ~ m i c e ~(S t o c k ~ N o: ~ 021,160, ~}$ The Jackson Laboratory) and homozygous B6.Cg-Gt(ROSA)26Sor ${ }^{\text {tm14 }}$ (CAG-tdTomato)Hze/J mice (Stock No: 007914, The Jackson Laboratory) were kept in a $12 \mathrm{~h}$ day/night cycle with access to food and water ad libitum. White light ( $300 \pm 50$ lux) was present from 7 a.m. to 7 p.m. and no light from 7 p.m. to 7 a.m. Homozygous B6.129P2(Cg)-Cx3 $\mathrm{cr}^{\text {tm2.1 }}$ (cre/ERT2)Litt/WganJ mice and homozygous B6.Cg-Gt(ROSA)26Sor ${ }^{\text {tm14 }}$ (CAG-tdTomato)Hze $/ \mathrm{J}$ mice were crossed to obtain heterozygous mice bearing a tamoxifen-inducible expression of the tandem dimer Tomato fluorescent in microglia in the brain. Mice were injected with tamoxifen dissolved in corn oil $\left(75 \mathrm{mg} \mathrm{kg}^{-1}\right)$ to induce the expression at postnatal day 35 . Surgery was performed between 2 weeks and 1 month after.

\subsection{Surgical implantation}

Mice were anesthetised with isoflurane inhalation (induction 0.8-1.5 $1 \mathrm{~min}^{-1}, 4-5 \%$; maintenance $0.8-1.51 \mathrm{~min}^{-1}, 1-2 \%$ ). Analgesia was performed by subcutaneous injection of buprenorphine $(0.1 \mathrm{mg}$ $\left.\mathrm{kg}^{-1}\right)$, and a local subcutaneous injection of lidocaine $\left(6 \mathrm{mg} \mathrm{kg}{ }^{-1}\right)$ and bupivacaine $\left(2.5 \mathrm{mg} \mathrm{kg}^{-1}\right)$ with a 1:1 ratio. The depth of anaesthesia was assessed with the pedal reflex, and artificial tears were used to prevent 
the eyes from drying. The temperature was maintained at $37^{\circ} \mathrm{C}$ with a heating pad during both surgical procedures and recording sessions. The skin of the head was shaved and cleaned with betadine. Mice were then placed on a stereotaxic frame, and the skin was opened to expose the skull. A squared craniotomy of approximately $(4 \times 4 \mathrm{~mm})$ was opened over the visual cortex (identified by stereotaxic coordinates), and the dura mater was removed. UV-sterilised all-polymeric TNPs were inserted in the cortex using a micromanipulator (SM-15R, Narishige). The probes were always implanted with the PEDOT:PSS:EG layer exposed to the caudal part of the brain to clearly discriminate the two sides during image analysis. A reference screw electrode was implanted in the rostral side of the cranium. The craniotomy was closed using dental cement, and an extra layer of dental cement was applied to secure the probe. The mice were left to recover on a heating pad and later returned to their cage.

\subsection{Acute induction of seizure and monitoring of epileptic activity}

Immediately after surgery, mice were removed from the stereotaxic apparatus while still under general anaesthesia, a needle electrode was placed subcutaneously in the dorsal area near the tail as ground, and the four channels of all-polymeric TNPs and the reference electrode were connected to the amplifier (BM623, Biomedica Mangoni). The signals were recorded using the WinAver software (Biomedica Mangoni). The recording was started (bandpass filtered $0.1-2000 \mathrm{~Hz}$ and sampling frequency $8192 \mathrm{~Hz}$ ) and, after a baseline period, $20 \mu \mathrm{l}$ of pentylenetetrazol (PTZ; $50 \mathrm{mg} \mathrm{ml}^{-1}, 45 \mathrm{mg} \mathrm{kg}^{-1}$ ) were delivered via intraperitoneal injection.

\subsection{Chronic recordings}

A first acute recording session was carried out immediately after the implantation to check the functionality of the TNPs at the starting point of the experiment. Chronic recordings were then performed 1- and 2weeks post-implantation and then every 2 weeks until 12 weeks. The mice were dark-adapted for $2 \mathrm{~h}$ before each chronic recording session. Mice were anaesthetised with a mixture of ketamine $\left(87.5 \mathrm{mg} \mathrm{kg}^{-1}\right)$ and xylazine $\left(12.5 \mathrm{mg} \mathrm{kg}^{-1}\right)$. The depth of anaesthesia was assessed with the pedal reflex, and artificial tears were used to prevent the eyes from drying. The temperature was maintained at $37{ }^{\circ} \mathrm{C}$ with a heating pad during both surgical procedures and recording sessions. Mice were placed on a stereotaxic apparatus in front of a Ganzfeld flash stimulator (BM6007IL, Biomedica Mangoni) and a needle electrode was placed subcutaneously in the dorsal area near the tail as ground. The four channels of all-polymeric TNPs and the reference electrode were connected to the amplifier (BM623, Biomedica Mangoni). The signals were recorded using the WinAver software (Biomedica Mangoni). First, a baseline recording was obtained for $2.5 \mathrm{~min}$ (bandpass filter 0.1-2000 $\mathrm{Hz}$ and sampling frequency $8192 \mathrm{~Hz}$ ). The recording noise was extracted from the baseline recording using a moving average window of 10 points; the mean \pm s.d. was calculated for each $500 \mathrm{~ms}$ epoch, then the mean s.d. of all the epochs was averaged to obtain the noise of the whole recording. Channels with a noise level higher than the average plus twice the s.d. of the noise at each time point were considered as nonworking channels and excluded from further analysis. Local field potentials (LFPs) were then acquired for $2.5 \mathrm{~min}$ (bandpass filter 0.1-100 $\mathrm{Hz}$ and sampling frequency $819.2 \mathrm{~Hz}$ ). After the recordings, a high pass filter at $0.5 \mathrm{~Hz}$ was applied, and the periodogram was calculated using the Welch method (window of $4 \mathrm{~s}$ ) on the whole recording. The area below the curve between 0.5 and $4 \mathrm{~Hz}$ frequencies (corresponding to the delta band) was approximated using the composite Simpson's rule. The power of the delta band was then divided by the total power (area below the periodogram) to obtain the relative power of the delta band. Last, visually evoked potentials were recorded (bandpass filter $0.1-200 \mathrm{~Hz}$ and sampling frequency $2048 \mathrm{~Hz}$ ) upon the presentation of 10 consecutive flashes $\left(4 \mathrm{~ms}, 10 \mathrm{~cd} \mathrm{~s} \mathrm{~m}^{-2}\right)$ delivered at $1 \mathrm{~Hz}$ of repetition rate. Data were processed and analysed using MATLAB (MathWorks). After each session, the mice were left to recover on a heating pad and later returned to their cage.

\subsection{Euthanasia}

Animals were euthanised with an injection of pentobarbital (150 mg $\mathrm{kg}^{-1}$ ) under a chemical hood. The chest cavity was opened to expose the beating heart, and a needle was inserted in the left ventricle, while the right atrium was cut to allow complete bleeding. The animal was immediately perfused with PBS followed by a fixative solution of $4 \%$ paraformaldehyde (PFA) in PBS. At the end of the procedure, the head of the animal was removed, and the brain collected and placed in 4\% PFA overnight for post-fixation.

\subsection{Histological analysis}

Brain samples were cryoprotected in sucrose $30 \%$ and frozen in optimal cutting temperature compound. $20-\mu \mathrm{m}$ thick horizontal sections of the brain were obtained using a cryostat (Histocom, Zug, Switzerland) and placed on microscope slides. The sections were washed in PBS, permeabilised with PBS + Triton $0.1 \%$ (Sigma-Aldrich), left for $1 \mathrm{~h}$ at room temperature in blocking buffer (Triton $0.1 \%+5 \%$ normal goat serum), and incubated overnight at $4{ }^{\circ} \mathrm{C}$ with primary antibodies for the glial fibrillary acidic protein (GFAP; 1:1000; Z0334, Dako), the cluster of differentiation 68 protein (CD68; 1:400; MCA1957, Biorad) and neural nuclei (NeuN; 1:500; ABN90P, Millipore). The day after, the sections were incubated for $2 \mathrm{~h}$ at room temperature with secondary antibodies (1:500; Alexa Fluor 647 and 488, Abcam), counterstained with DAPI (1:300; Sigma-Aldrich) and mounted for imaging with Fluoromount solution (Sigma-Aldrich). Representative images were acquired with a confocal microscope (LSM-880, Zeiss). For image segmentation and quantification, images were acquired using a slide scanner microscope (VS120, Olympus; $20 \times$ objective, pixel size $0.34 \mu \mathrm{m}$ ) and analysed in Python, using the scikit-image package (https://scikit-image.org/).

\subsection{Whole-brain imaging}

Brain clarification was performed according to a previously described procedure [20]. Briefly, after overnight post-fixation at $4{ }^{\circ} \mathrm{C}$ in PFA 4\%, the brain was immersed in hydrogel solution (Acrylamide $40 \%$ + VA-044 initiator powder in PBS) at $4{ }^{\circ} \mathrm{C}$ for three days. The hydrogel polymerization was induced by keeping the sample at $37{ }^{\circ} \mathrm{C}$ for $3 \mathrm{~h}$. Then, the brain was passively clarified in $4 \%$ sodium dodecyl sulfate clearing solution (pH 8.5) for four weeks under gentle agitation at $37^{\circ} \mathrm{C}$. The whole clarified brain was transferred to Histodenz solution at $\mathrm{pH} 7.5$ (Sigma-Aldrich). Brains were immersed in a refractive index matching solution (RIMS) containing Histodenz for at least $24 \mathrm{~h}$ before being imaged. Brains were glued to a holder and immersed in a $10 \times 20 \times 45$ $\mathrm{mm}^{3}$ quartz cuvette filled with RIMS. The cuvette was then placed in a chamber filled with oil with $(\mathrm{n}=1.45$; Cargille). A custom-made light-sheet microscope optimised for labelled clarified tissue was used to image the implant within the mouse brain (COLM, clarity optimised light-sheet microscope [21]). The sample was illuminated (488 and 554 $\mathrm{nm}$ ) by two digitally scanned light-sheets coming from opposite directions, and the emitted fluorescence was collected by high numerical aperture objectives (Olympus XLPLN10XSVMP, N.A 0.6) filtered (Brightline HC 525/50, Semrock) and imaged on a digital CMOS camera (Orca-Flash 4.0 LT, Hamamatsu) at a frequency ranging between 5 and 10 frames per second. A self-adaptive positioning of the light sheets across Z-stacks acquisition ensured an optimal image quality over up to $1 \mathrm{~cm}$ of tissue. To acquire images of whole samples at lower resolution another custom-made light-sheet microscope was used (mesoSPIM, mesoscale selective plane illumination microscopy [22]). The microscope consists of a dual-sided excitation path using a fibre-coupled multiline laser combiner (405, 488, 561 and $647 \mathrm{~nm}$; Toptica MLE) 
and a detection path comprising a 42 Olympus MVX-10 zoom macroscope with a $1 \times$ objective (Olympus MVPLAPO), a filter wheel (Ludl 96A350), and a CMOS camera (Orca Flash 4.0 V3, Hamamatsu). The excitation paths also contain galvo scanners for light-sheet generation and reduction of shadow artefacts due to absorption of the light-sheet. In addition, the beam waist is scanned using electrically tuneable lenses (EL-16-40-5D-TC-L, Optotune) synchronised with the rolling shutter of the CMOS camera. This axially scanned light-sheet mode leads to a uniform axial resolution across the field-of-view of $5 \mu \mathrm{m}$. Image acquisition was made using custom software written in Python. Z-stacks were acquired at $3 \mu \mathrm{m}$ spacing with a zoom set at $2 \mathrm{x}$ resulting in an in-plane pixel size of $7.8 \mu \mathrm{m}(2048 \times 2048$ pixels $)$. The excitation wavelength was set at $561 \mathrm{~nm}$ with an emission $530 / 40 \mathrm{~nm}$ bandpass filter (BrightLine HC, AHF).

\subsection{Statistical analysis and graphical representation}

Statistical analysis and graphical representation were performed

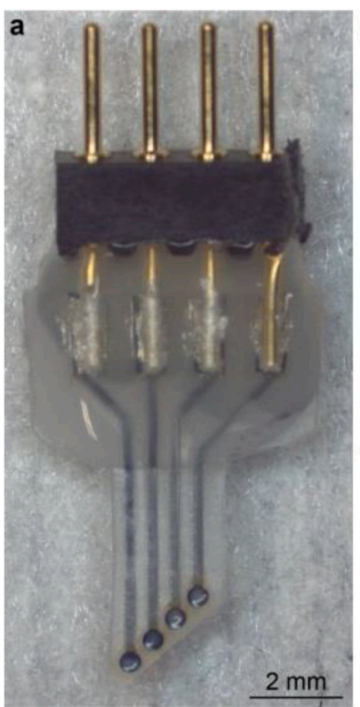

f

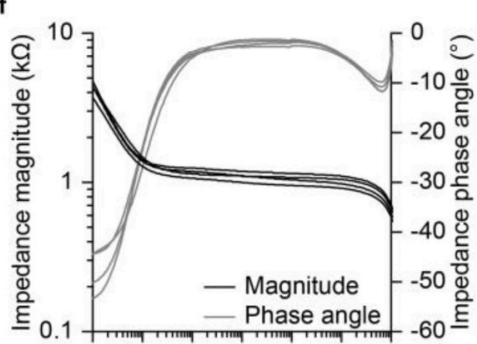

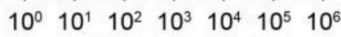
Frequency $(\mathrm{Hz})$

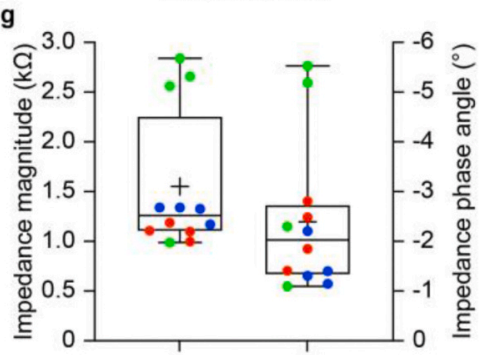

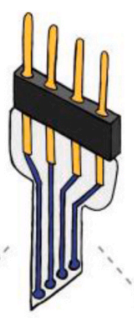

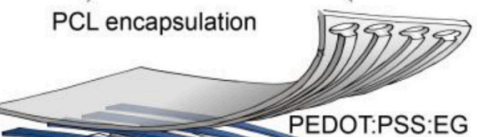

PCL substrate

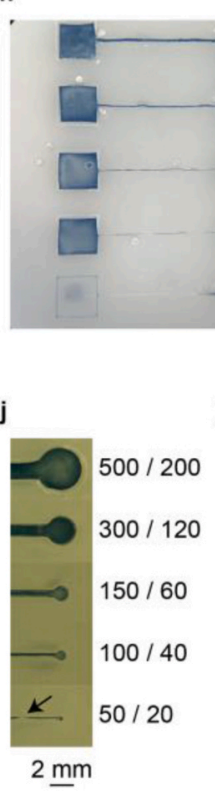

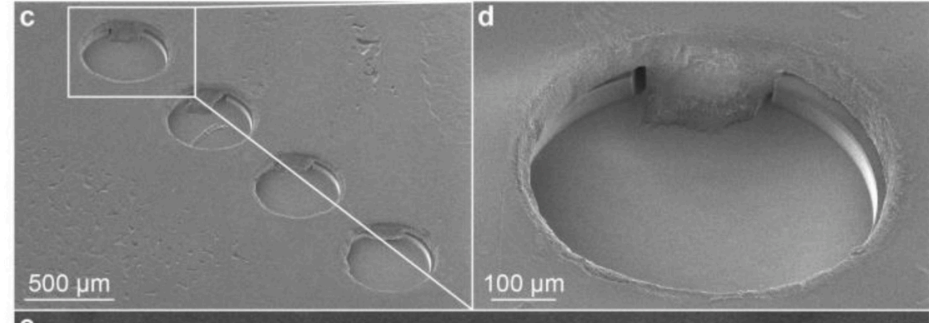

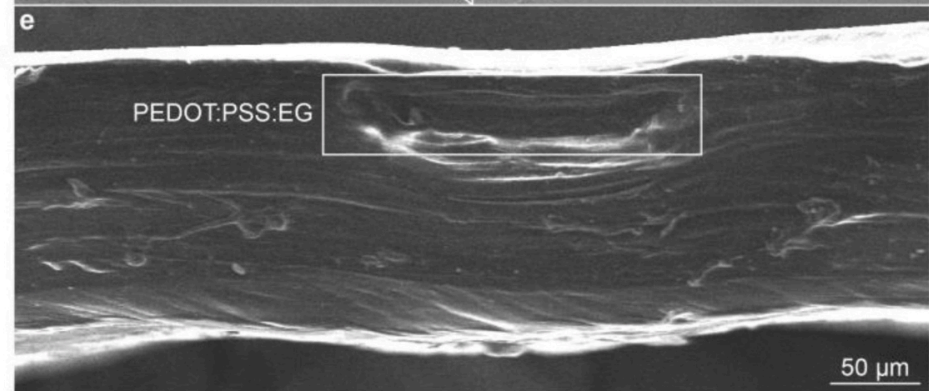

i

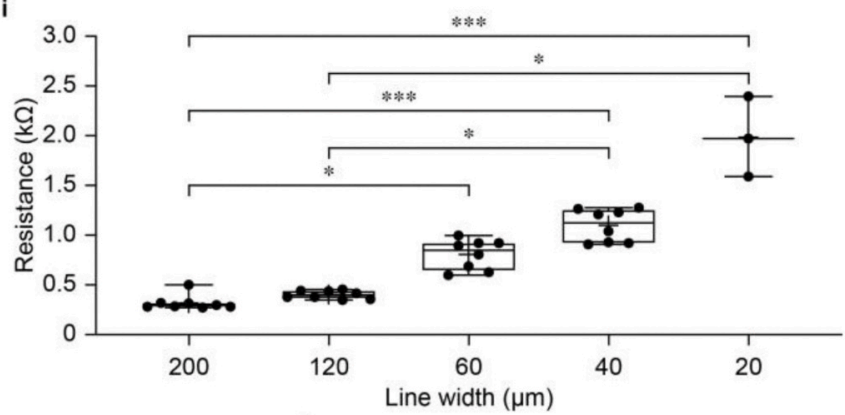

$$
2 \underline{\mathrm{mm}}
$$

k

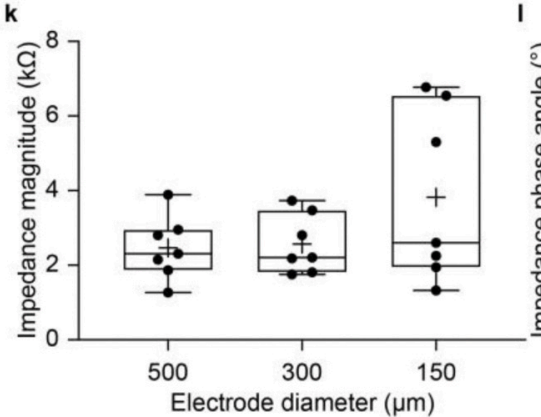

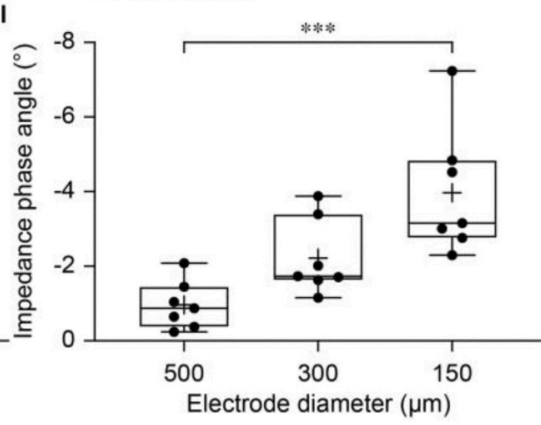

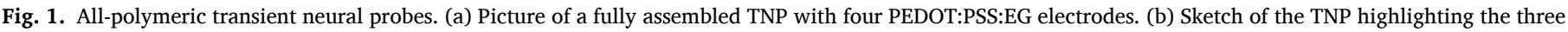

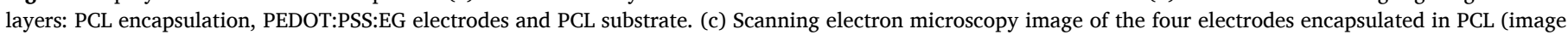

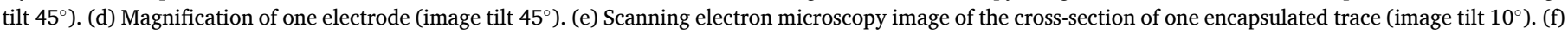

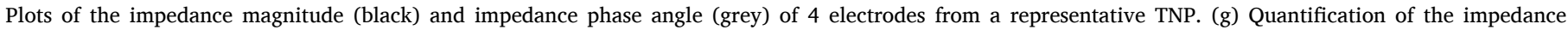

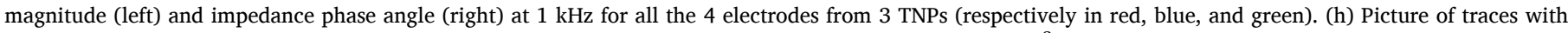

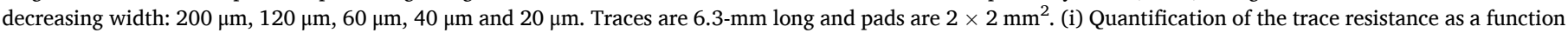

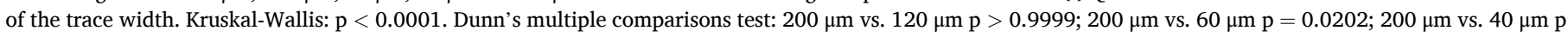

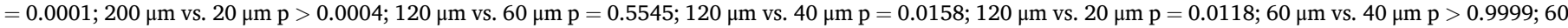

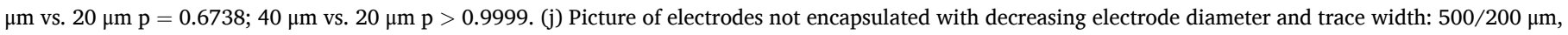

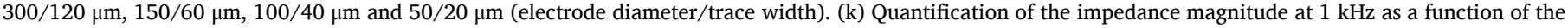

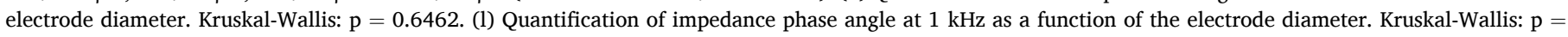

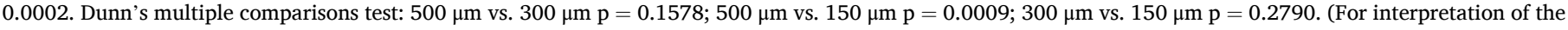
references to colour in this figure legend, the reader is referred to the Web version of this article.) 
with Prism 8 (Graph Pad). The normality test (D'Agostino \& Pearson omnibus normality test) was performed in each dataset to justify the use of a parametric or non-parametric test. The box plots always extend from the 25 th to 75 th percentiles. The line in the middle of the box is plotted at the median. The + is the mean. The whiskers go down to the smallest value and up to the largest. In each figure p-values were represented as: *p $<0.05, * * \mathrm{p}<0.01, * * * \mathrm{p}<0.001$, and $* * * * \mathrm{p}<0.0001$.

\section{Results}

\subsection{Probe fabrication and characterization}

We fabricated TNPs based on PCL as substrate and encapsulation layers, and PEDOT:PSS doped with EG as a conductive element, by using soft lithographic techniques (Fig. $1 \mathrm{a}$ and b and Figs. 1). PCL is a commercial biodegradable polyester widely used in biomedical applications [23-26]. It is easy to process and has a longer degradation time (several months or years [23]) compared to most biodegradable polymers. PEDOT:PSS dispersed in water is commercially available, has excellent biocompatibility, has the ability to support cells viability $[27,28]$, and can be integrated into softer systems like elastomers [29,30] and hydrogels [31,32]. EG doping of PEDOT:PSS is known to improve its conductivity [33,34]. The micro-structured PCL-based substrate (thickness 60-70 $\mu \mathrm{m}$ ) and encapsulation (thickness 60-70 $\mu \mathrm{m}$ ) layers have been obtained by replica moulding. The hollow regions of the substrate layer have been manually filled with PEDOT:PSS:EG to create electrodes, traces and contact pads (Figs. 1). Electrodes are $500-\mu \mathrm{m}$ in diameter (Fig. 1c and d) and traces are $200-\mu \mathrm{m}$ wide (Fig. 1e). At the electrode level, the PEDOT:PSS:EG layer is approximately $3-\mu \mathrm{m}$ thick, while at the trace level, it is approximately $1-\mu \mathrm{m}$ thick (Figs. 2). After curing of the PEDOT:PSS:EG, the PCL encapsulation layer has been manually flip-bonded to the substrate layer via a low-temperature process (total probe thickness ranging from 120 to $140 \mu \mathrm{m}$ ). This procedure ensured a complete filling of the trenches for the conductive traces (Fig. 1e). IS showed remarkable performances of all-polymeric TNPs based on PEDOT:PSS:EG, characterised by low impedance magnitude (Fig. 1f) and low impedance phase angle (Fig. 1g) over a wide range of frequency (from $10 \mathrm{~Hz}$ to $100 \mathrm{kHz}$ ), which makes them appropriate for the recording of both low- and high-frequency neuronal signals, such as LFPs and neural spiking activities respectively. The comparison of multiple probes showed very low intra-probe variability. On the other hand, the inter-probe variability might be explained by the manual deposition process of PEDOT:PSS:EG resulting in a variable thickness of the conductive layer. To verify that the excellent electrochemical characteristics of the TNPs were not related to shortcuts between electrodes caused by the porosity of the PCL scaffold, we took advantage of an all-polymeric TNP with a modified design embedding 17 electrodes (Figs. 3). In this specific device, four traces were interrupted, due to a gap in the PEDOT:PSS:EG layer (Figs. 3a-c). As expected, only the corresponding electrodes were rightfully not functional (Figs. 3d and e), thus confirming that shortcuts between electrodes are not present. Besides electrochemistry, we also measured the averaged line resistance of the traces, which was $319.5 \pm 75.01 \Omega( \pm$ s.d., $n=8$ traces; Fig. 1 h and i) for $200-\mu \mathrm{m}$ wide and $6.3-\mathrm{mm}$ long traces.

Then, we investigated the minimum dimensions for electrodes and traces that could be reached using these materials and fabrication methods. First, we fabricated narrow traces and measured the average line resistance ( $n=8$ traces for each dimension; Fig. 1 h and i). As expected, the line resistance significantly increased with the decrease of the width. It must be noted that for 20 - $\mu$ m wide traces, only 3 out of 8 traces appeared to be connected. Therefore, the resolution limit for traces is $40 \mu \mathrm{m}$. Next, we fabricated TNPs with smaller electrodes and traces (Fig. $1 \mathrm{j}$ ) and tested IS ( $n=7$ electrodes for each dimension). As expected, the impedance magnitude (Fig. 1k) and the impedance phase angle (Fig. 11) increased with the reduction of the electrode diameter. Among the dimensions tested, the smallest electrode $(50-\mu \mathrm{m}$ electrode diameter and $20-\mu \mathrm{m}$ trace width) resulted not connected (Fig. $1 \mathrm{j}$, black arrow), as expected due to the narrow trace. Electrodes with $100-\mu \mathrm{m}$ dimeter ( $40-\mu \mathrm{m}$ trace width) resulted not working because the electrode openings in the PCL encapsulation layer closed during the bonding to the PCL substrate. Hence, the minimum dimensions reached for the electrode diameter was $150 \mu \mathrm{m}(60-\mu \mathrm{m}$ trace width).

Pure PEDOT:PSS electrodes have been previously described for invitro applications to record cardiac and neural signals and in-vivo neuromodulation. A flexible all-polymer microelectrode array reported impedance magnitude value ( $1.5 \mathrm{M} \Omega)$ at $1 \mathrm{kHz}$ for $120-\mu \mathrm{m}$ electrodes substantially higher than the ones reported here [35]. The higher impedance magnitude was caused by the deposition method of PEDOT: PSS in a polymeric channel, which led to surface coating only and not to the complete filling of the channel, thus reducing the electrode surface area. A second device exploring inkjet-printed PEDOT:PSS reported an impedance magnitude value $(19.5 \mathrm{k} \Omega$ ) at $1 \mathrm{kHz}$ for an electrode area corresponding approximately to the area of a round electrode with a diameter of $300 \mu \mathrm{m}$ comparable to the TNP [36]. In a third report, PEDOT:PSS/glycerol electrodes showed an average impedance magnitude at $1 \mathrm{kHz}$ of $6.3 \mathrm{k} \Omega$ for an electrode area of $0.5 \mathrm{~mm}^{2}$ (corresponding to the area of a round electrode with a diameter of $800 \mu \mathrm{m}$ ) which is similar to the TNP [37].

Last, cytotoxicity tests performed in-vitro showed a cell viability of $96.9 \pm 7.2 \%$ (mean \pm s.d., 2 probes, 6 replicas per probe) and opened the way for the in-vivo validation of the devices (Fig. 2).

\subsection{Functional validation in-vivo}

To assess the capability of all-polymeric TNPs in recording neural activity, we performed in-vivo acute brain recordings in mice upon induction of epileptic seizures. All-polymeric TNPs were implanted in the visual cortex area of anaesthetised mice (Fig. 3a), and the neural activity was recorded both before and after intraperitoneal injection of the convulsant PTZ, which is routinely used to test anticonvulsants in animals [38-40]. The transition between resting state and epileptic activity after PTZ injection was clearly detected by the TNPs with an excellent signal to noise ratio (Fig. 3b), thus demonstrating the potential of these all-polymeric TNPs for monitoring epileptic activity.

Next, we performed chronic in-vivo recordings of baseline activity, LFPs at rest and visually evoked potentials (VEPs) to assess the longevity of the device. All-polymeric TNPs were implanted in the visual cortex area of mice, and their functionality was evaluated through periodic recordings up to 3 months post-implantation (Fig. 4). The broad-band noise of recordings was computed from baseline recordings $(2.5 \mathrm{~min}$, bandpass filter $0.1-2000 \mathrm{~Hz}$ and sampling frequency $8192 \mathrm{~Hz}$ ). The noise plot (Fig. 4a) shows the evolution of the noise level for each working electrode over time (filled circles). Once an electrode was found not properly functional (empty circles), it was excluded from further analysis. The applied criteria of exclusion were the following: i) appearance of periodic artefacts not linked to any biological function (red arrow in Fig. 4a); ii) noise level higher than the average plus twice the standard deviation of the noise at each time point; iii) lack of VEPs. The number of excluded electrodes progressively increased over time (Fig. 4b), with 3 out of 16 electrodes still properly functional after three months. LFPs (2.5 $\mathrm{min}$, bandpass filter $0.1-100 \mathrm{~Hz}$ and sampling frequency $819.2 \mathrm{~Hz}$ ) are not strongly affected by broad-band noise because of the low pass filter at $100 \mathrm{~Hz}$. On the other hand, LFPs are characterised by a strong delta rhythm $(0.5-4 \mathrm{~Hz})$ due to the animal anaesthesia (Fig. 4c). Early after implantation, electrodes showed a narrow power spectral density (PSD) in the range of delta rhythm (black traces in Fig. 4c and black lines in Fig. 4d). Over time, because of the electrode deterioration, the normalised PSD shows activity at higher frequency in electrodes that were previously considered not properly functional because of high broad-band noise (grey line in Fig. 4d). Conversely, electrodes still considered functional showed a narrow PSD (red line in Fig. 4d). As a consequence, the relative power of the delta band 


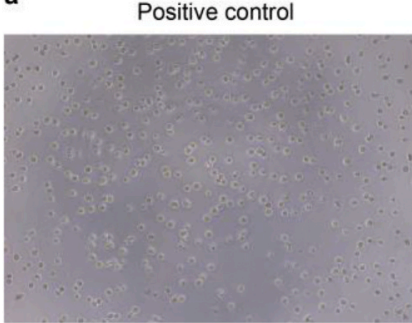

Negative control

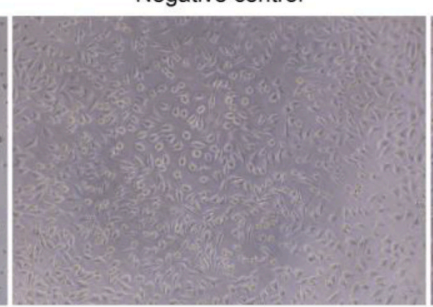

TNP

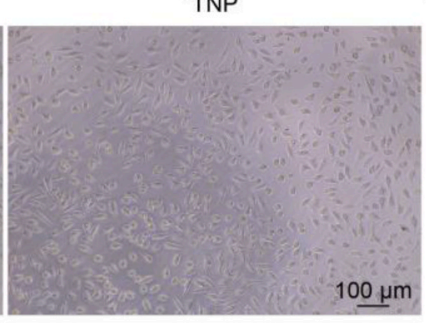

b

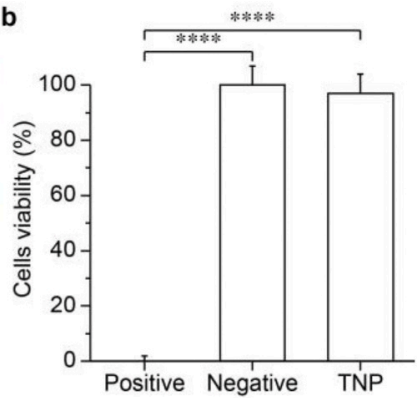

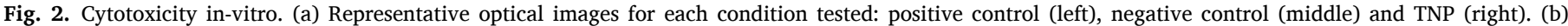

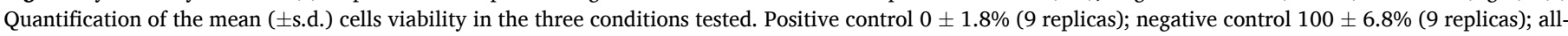

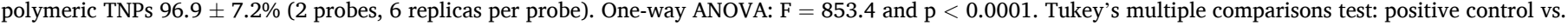
negative control $\mathrm{p}<0.0001$; positive control vs. neural probes $\mathrm{p}<0.0001$; negative control vs. neural probes $\mathrm{p}=0.4852$.

a
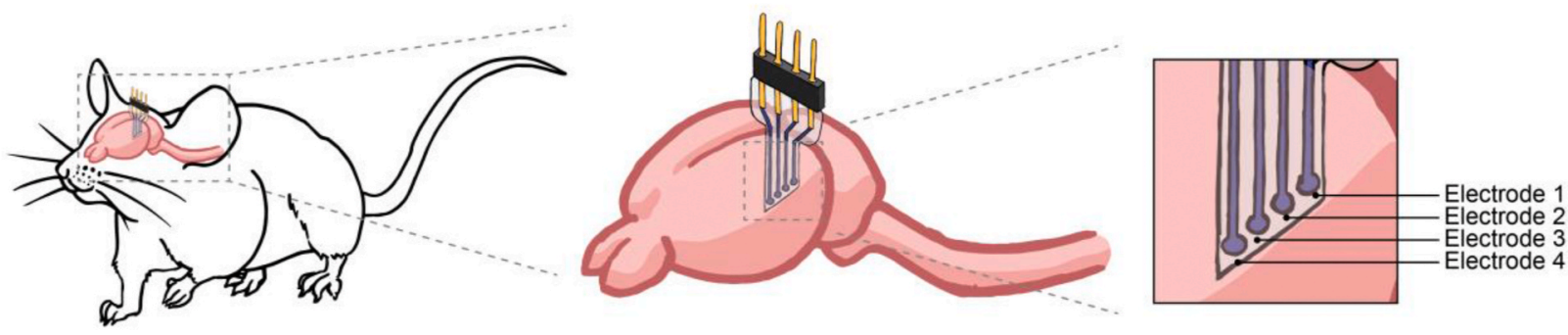

b

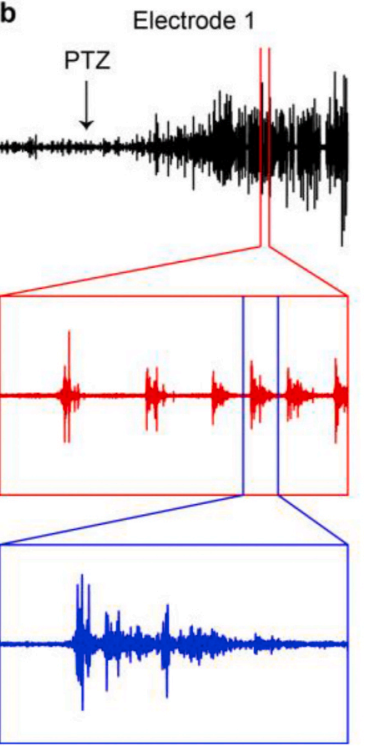

Electrode 2

Electrode 3
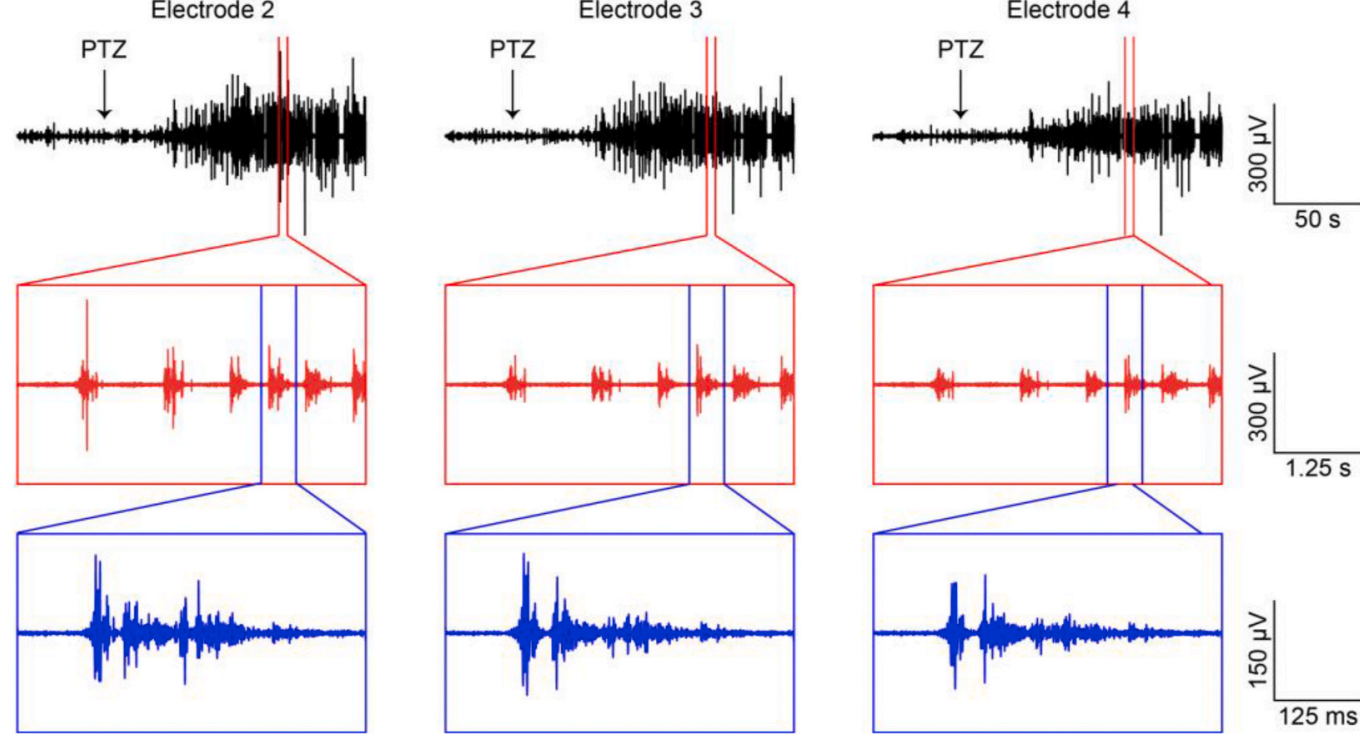

Fig. 3. Acute recordings with all-polymeric transient neural probes. (a) Sketch of the TNP implanted in a mouse brain for in-vivo recordings. (b) Detection of epileptic activity after injection of PTZ (black arrow) from the four electrodes of a all-polymeric TNP. The red and blue boxes show an enlargement of the recorded activity. (For interpretation of the references to colour in this figure legend, the reader is referred to the Web version of this article.)

decreases with the deterioration of the electrodes (Fig. 4e). For each electrode, early after implantation, a low noise correlates with a high relative power of the delta band. However, at the exclusion point, there is a reduction of the relative delta power which is globally proportional to the increase of noise (linear regression: slope $=-1.369$, $\mathrm{R}$ square $=$ 0.527). Overall, all-polymeric TNPs retain good in-vivo recording capabilities for months after implantation, even if they progressively lose functionality and only a few electrodes are still properly functional after three months.

At this stage, the cause of electrode deterioration is not easy to determine. So far, only a few studies have investigated the chronic performances of PEDOT:PSS in-vivo [41-44], and the results indicated a reduction in performance after a few months of implantation. Many factors could contribute to the deterioration of PEDOT:PSS in-vivo, related to both materials properties and interaction with the biological environment. A commonly reported problem is the adhesion of PEDOT: PSS to the substrate [45]. The weak adhesion between PEDOT:PSS and polyesters [46], like PCL, plays a crucial role since PEDOT:PSS might be subjected to water-induced swelling and delamination during the degradation of the PCL scaffold. PEDOT has also been reported to undergo degradation by hydrolysis when exposed to salt aqueous solutions [47] or by high concentration of hydrogen peroxide, which is a physiological oxidant [48,49]. Macrophages cultured with PEDOT:PSS generated a significant amount of hydrogen peroxide, that is a significant factor involved in cellular phagocytosis [50]. PEDOT:PSS could possibly be degraded by the combination of hydrolysis and presence of 


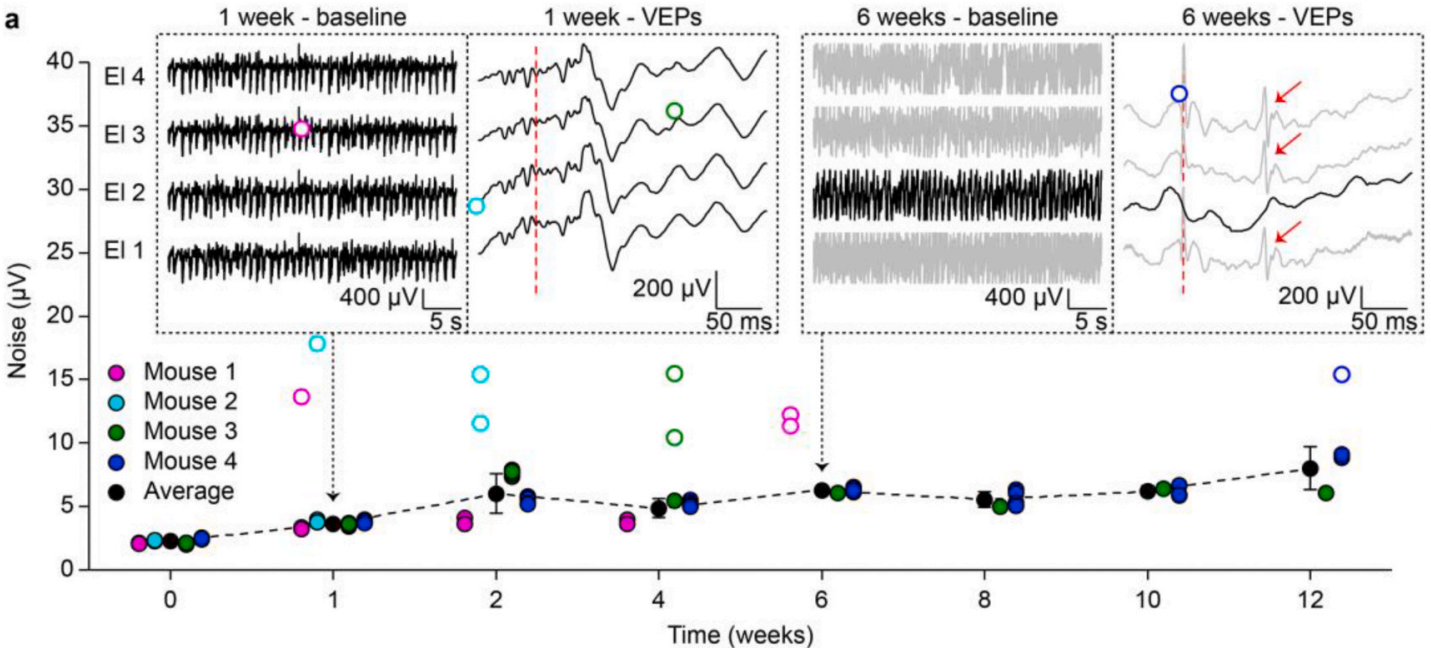

c
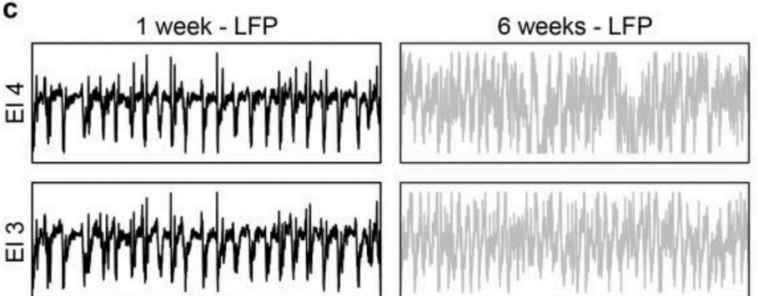

d
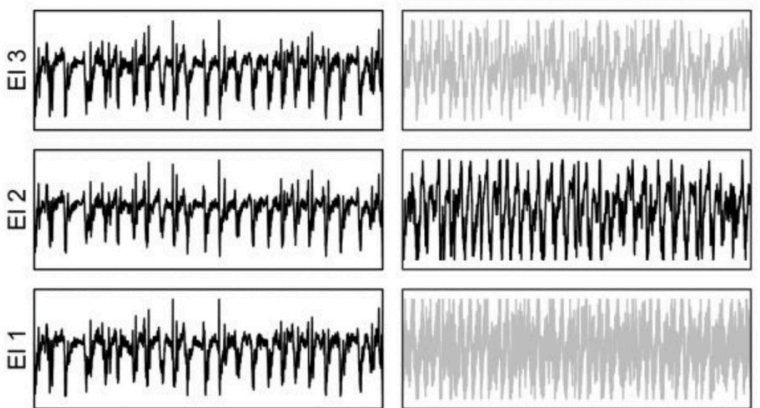

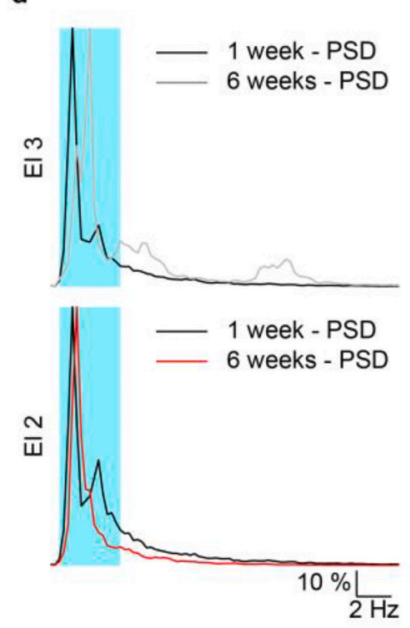

e

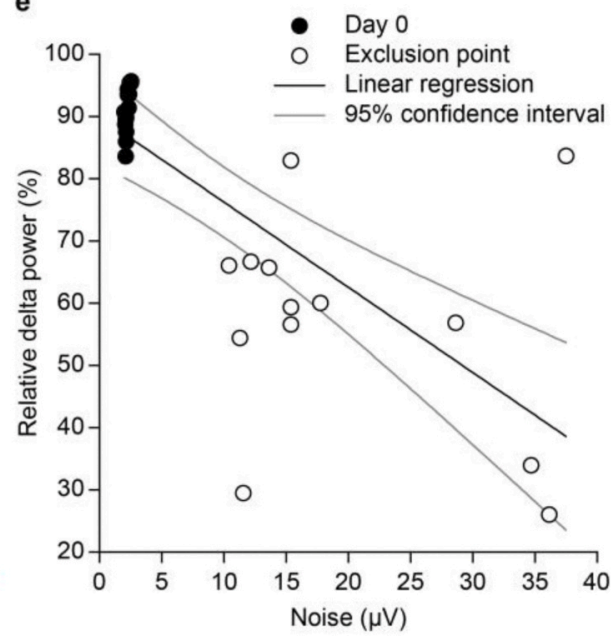

Fig. 4. Long-term functioning in-vivo. (a) Noise evaluation as a function of time during in-vivo chronic recordings at fixed time points. Different colours correspond to different mice; filled circles correspond to the electrodes considered functional and thus included in the calculation of the average ( \pm s.d.) noise values (black circles and dashed line), while empty circles correspond to the electrodes considered not correctly working and thus excluded from the calculation of the average noise. The two insets show single baseline recordings (left) and VEPs (right) from all the four electrodes of the TNP implanted in mouse 3 at week 1 and week 6 postimplantation; the red dashed line indicates the light stimulus for VEPs $\left(10 \mathrm{~cd} \mathrm{~s} \mathrm{~m}^{-2}\right)$. The traces in grey are from electrodes considered not functional. The red arrows show periodic artefacts not linked to any biological function. (b) Plot of the percentage of working electrodes as a function of time during in-vivo chronic experiments. (c) Representative example of LFPs recorded from all the electrodes of the TNP implanted in mouse 3 at week 1 and week 6 post-implantation. The traces in grey are from electrodes considered not functional. (d) Representative examples of normalised power spectral densities obtained from the recordings from two electrodes (numbers 2 and 3 ) of the probe implanted in mouse 3 at week 1 (black traces) and week 6 (black grey and red) after implantation. The trace in grey is from the electrode considered not functional, while the trace in red is from the electrode considered still functional. (e) Correlation between the relative power of the delta band and the noise of the electrodes immediately after implantation (16 electrodes, black circles) or at the time point of exclusion (13 electrodes, empty circles). The black line is the linear regression, while the grey lines the $95 \%$ confidence interval. (For interpretation of the references to colour in this figure legend, the reader is referred to the Web version of this article.)

hydrogen peroxide following device implantation [45].

\subsection{Evaluation of the foreign body reaction}

To investigate the acute phase of the foreign body reaction induced by TNPs, we performed a histological paired comparison between allpolymeric TNPs and polyimide (PI) implants, surgically placed in the two hemispheres of the brain of the same mice (Fig. 5a). The PI implants were chosen as a reference for non-degradable flexible implants. Also, the implants have been manufactured with similar dimensions to be more comparable (TNP: $\sim 130-\mu \mathrm{m}$ thick and $1.3-\mathrm{mm}$ wide; PI: $125-\mu \mathrm{m}$ thick and $0.7-\mathrm{mm}$ wide). Evaluations have been made at 1 - and 2months post-implantation, 6 mice for the first time point and 5 mice for the second.

For each mouse, horizontal brain slices were sampled at different cortical depths (Fig. 5a from 1 to 4) and analysed after image segmentation (Figs. 4). Images were rotated to align the probe horizontally (Figs. 4a) and converted to binary using a local threshold algorithm: all pixels which intensity was above the threshold were assigned the value of 1 , while to the rest of the pixels were assigned the value of 0 (Figs. 4b). A group of adjacent pixels with value 1 was defined as a blob, and the area of each blob was calculated, along with the coordinates of its centroid. The image was divided into $10 \times 10$ quadrants (Figs. 4c), for each of which the total area of blobs (with their centroid included in that quadrant) was computed. Then, the total area occupied by blobs in the zone adjacent to the probe (Figs. 4d) was calculated as the cumulative area of all the quadrants included in a rectangle defined by the extremities of the probe (manually specified for each of the images), minus the area of the probe itself. The percentage of the area occupied by blobs was computed as the area occupied by blobs over the proximal area. The average percentage of the proximal area occupied by the blobs for each sample was used as a parameter to compare the 
a

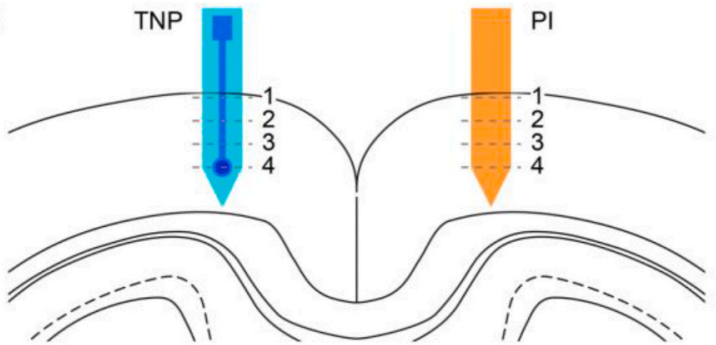

d
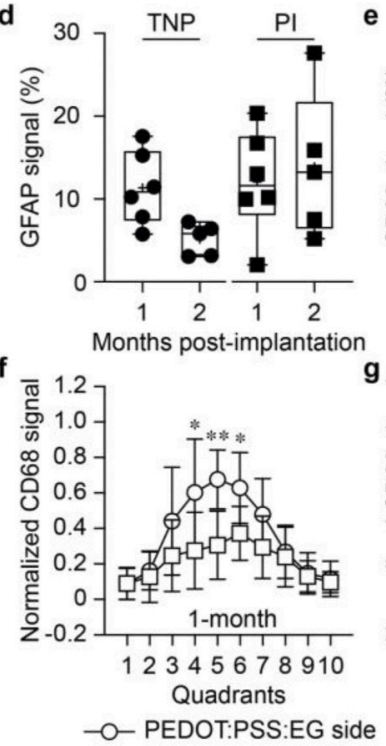

e $15, \quad$ TNP

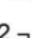

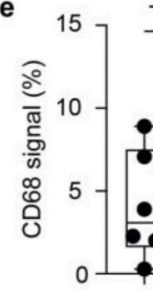

b
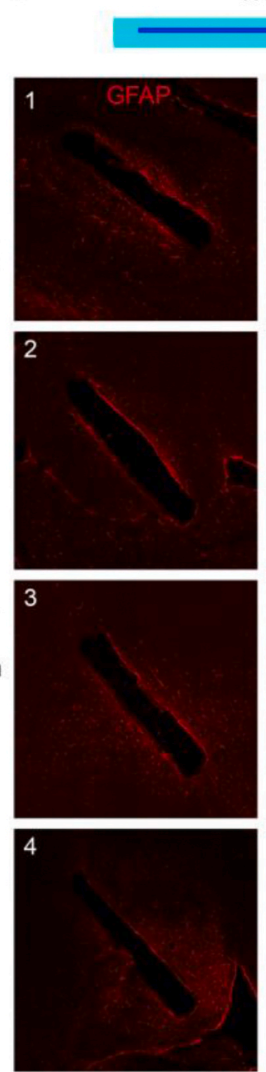

TNP
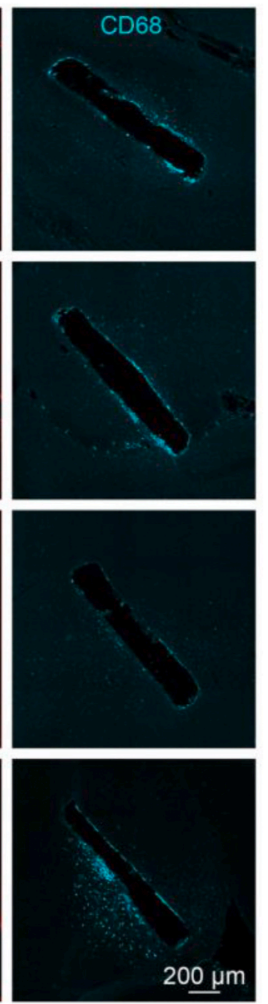
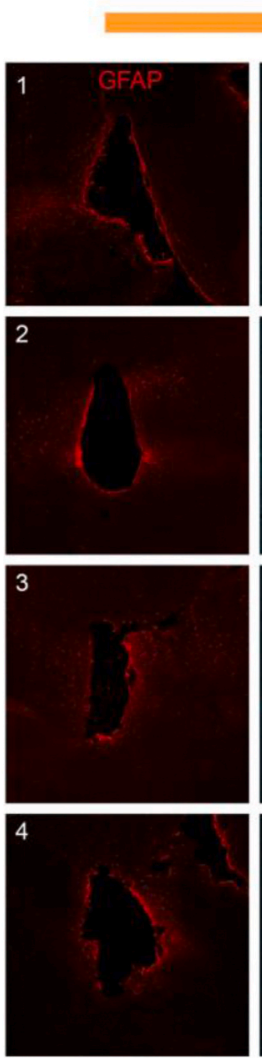

$\mathrm{PI}$
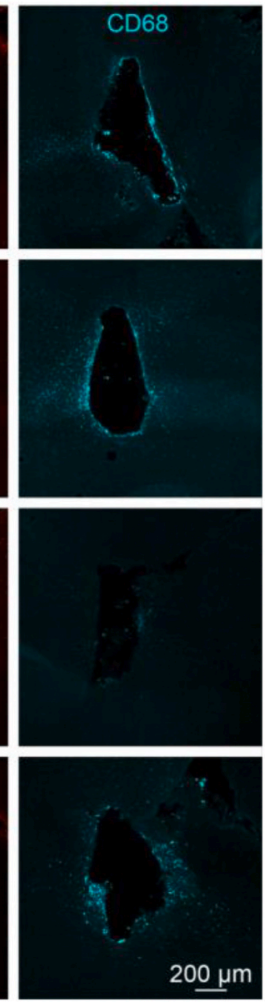

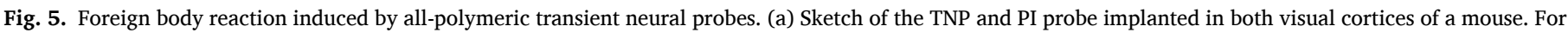

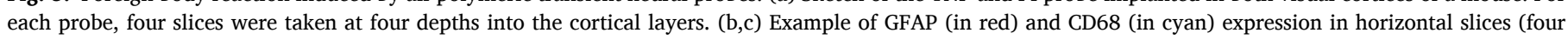

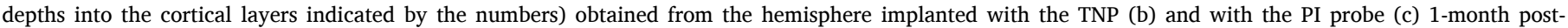

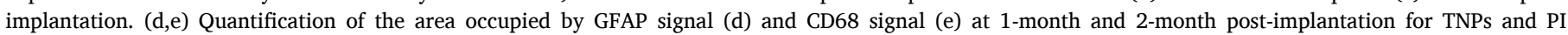

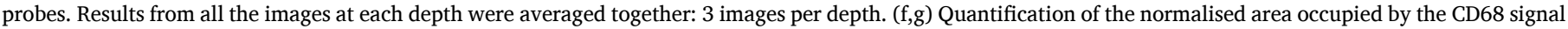

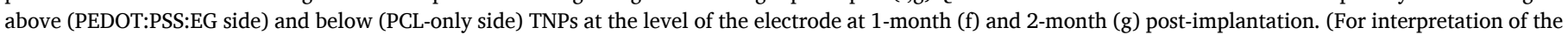
references to colour in this figure legend, the reader is referred to the Web version of this article.)

experimental groups. Three images for each depth were averaged. The staining for the GFAP (Fig. 5b and c) and the corresponding quantification analysis (Fig. 5d) performed on segmented images showed that the area occupied by the GFAP signal in the zone adjacent to the probe exhibits a decreasing trend over time for all-polymeric TNPs ( $\mathrm{p}=$ 0.0178 , two-tailed unpaired $t$-test) and a stable trend for PI probes $(\mathrm{p}=$ 0.6924 , two-tailed unpaired $t$-test). Also, the staining for CD68 (Fig. 5b, c), a marker of phagocytic structures in microglia [51], and the corresponding quantification analysis (Fig. 5e) showed that the area occupied by the CD68 signal exhibits a slightly increasing trend over time for all-polymeric TNPs ( $\mathrm{p}=0.2897$, two-tailed unpaired $t$-test), while it remains stable for PI probes ( $\mathrm{p}=0.5905$, two-tailed unpaired $t$-test).

Histological samples of brains implanted with TNPs showed a clustering of the CD68 signal at the level of the electrode (slice 4 in Fig. 5b and c), the only point where the PEDOT:PSS:EG is exposed to the tissue. Following this observation, we performed a second analysis where the segmented images were divided into two parts: the side of the probe with the PEDOT:PSS:EG exposed and the side of the probe with only the PCL exposed (Figs. 5). For each side, the cumulative area occupied by blobs was calculated along the horizontal axis and normalised by the global maximum. The probes were always implanted with the PEDOT: PSS:EG layer exposed to the caudal part of the brain to discriminate the two sides during image analysis. The analysis confirmed that the CD68 signal is highly clustered on the side of the PEDOT:PSS:EG electrodes at both time points (Fig. $5 \mathrm{f}$ and $\mathrm{g}$ ), suggesting an interaction between microglia and PEDOT:PSS:EG.
Next, we quantified the spatial extent of the GFAP and CD68 signals around the TNP at each cortical depth. Each image (one per depth) was rotated to align the probe vertically (Fig. 6a). Two regions of interest, one on the left and one on the right of the probe, were manually defined as rectangles perpendicular to the probe, spanning from the edge of the probe to the border of the image (Fig. 6b). For each of them, the average intensity along the horizontal axis was computed, starting from the edge of the probe to the border of the image. The average vector of intensities was normalised by dividing each point by the maximum value among the two regions of interest and a normalised intensity plot was generated (Fig. 6c). For each cortical depth, normalised intensity plots from all mice were averaged ( 6 mice at 1-month post-implantation and 5 mice at 2-months post-implantation) to determine the spatial profile. The spatial profiles of both GFAP (Fig. 6d and e) and CD68 (Fig. 6f and g) resulted uniform along the vertical axis of the probe (cortical depths) at both 1and 2-months post-implantation (Fig. 6d,f and Fig. 6e,g respectively). In order to quantify the extent of the GFAP and CD68 signals, we measured the distance from the probe at which the average profile among the 4 depths (black lines in Fig. 6d-g) is reduced by 50\% (red dots in Fig. $6 \mathrm{~d}-\mathrm{g}$ ). The quantification (Fig. $6 \mathrm{~h}$ ) revealed that the GFAP signal is wider 1-month post-implantation (116.82 and $206.16 \mu \mathrm{m}$ respectively for the left and the right side of the probe) and it is reduced 2-months post-implantation ( 96.55 and $95.18 \mu \mathrm{m}$ respectively for the left and the right side of the probe), although the reduction is not significant (p $=0.3333$, two-tailed Mann-Whitney test). This result is in agreement with the previous analysis (Fig. 5d). The CD68 signal is restricted to a 

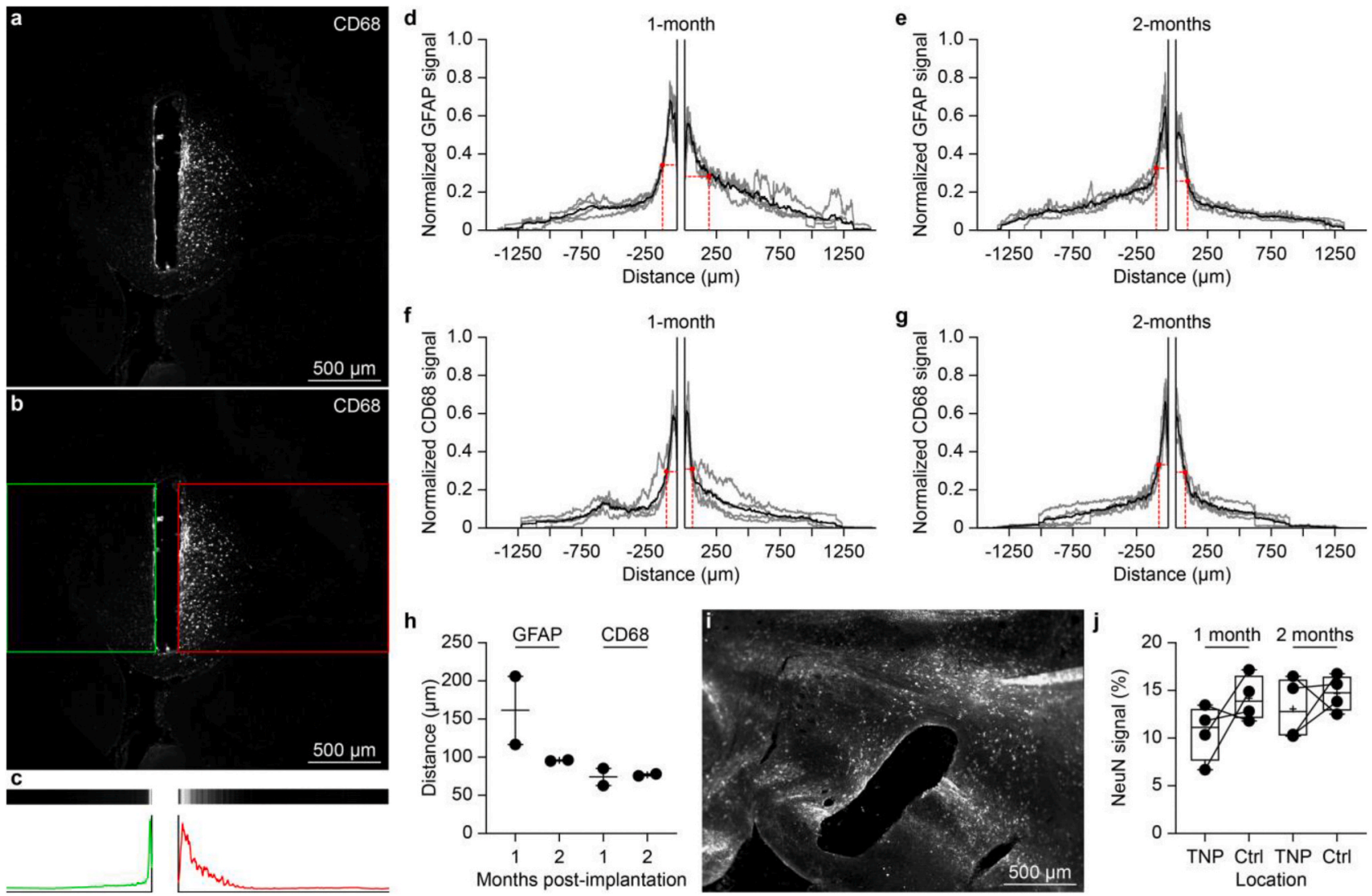

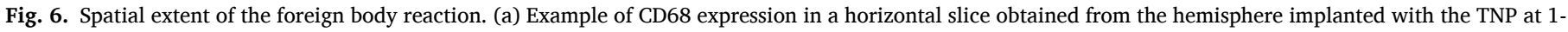

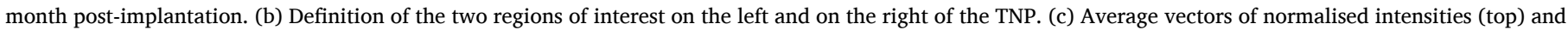

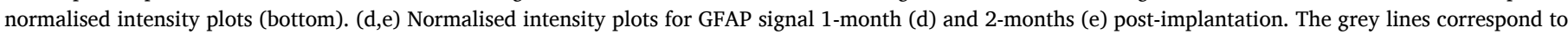

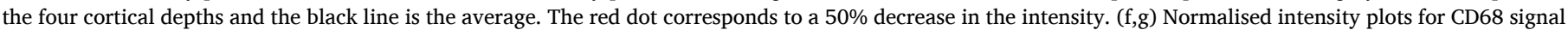

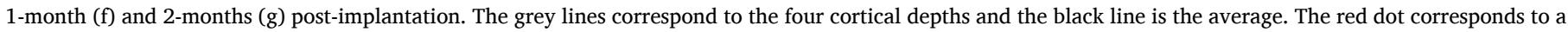

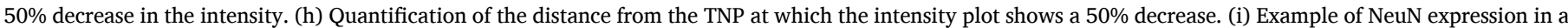

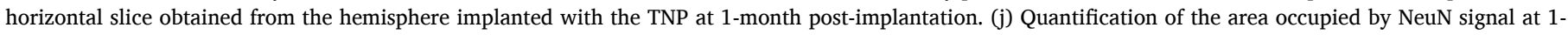

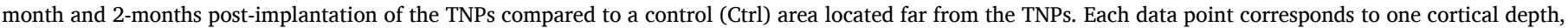
average of 3 images per depth. (For interpretation of the references to colour in this figure legend, the reader is referred to the Web version of this article.)

small area around the probe (1-month: 85.56 and $62.88 \mu \mathrm{m}$; 2-months: 75.60 and $78.00 \mu \mathrm{m}$; respectively for the left and the right side of the probe), and it does not change from 1- to 2-months post-implantation (Fig. 6h; p > 0.9999, two-tailed Mann-Whitney test), in agreement with the previous analysis (Fig. 5e).

We also quantified the area occupied by NeuN signal (a marker for neuronal cells) around the TNP at the four cortical depths (Fig. 6i) compared to a control area located far from the TNP. The quantification analysis (average of three sections per cortical depth), performed on one mouse per time point (1- and 2-months post-implantation), showed that the area occupied by the NeuN signal is not statistically different between the two areas (Fig. 6i; 1 -month: $\mathrm{p}=0.3750$, Wilcoxon matchedpairs signed rank test; 2-months: $\mathrm{p}=0.6250$, Wilcoxon matched-pairs signed rank test).

The clustering of the CD68 signal we observed at the level of the PEDOT:PSS:EG electrode (Fig. $5 \mathrm{f}$ and $\mathrm{g}$ ) encouraged us to further investigate the interaction between phagocytic microglial cells and PEDOT:PSS:EG. To this end, we fabricated TNPs with a PCL substrate coated with PEDOT:PSS:EG labelled with the fluorescent marker FITC [19] and implanted them into the cortex of mice expressing tdTomato in microglia (Fig. 7a). In this experiment, all-polymeric TNPs were not encapsulated to enhance interaction between microglial cells and PEDOT:PSS:EG. High-resolution imaging on clarified brains 1-month (Fig. 7b) and 2-months (Fig. 7c) post-implantation showed colocalization of PEDOT:PSS:EG (in green) and microglial cells (in red). This evidence suggests that the presence of PEDOT:PSS:EG detached from the PCL substrate stimulates phagocytic activity of microglial cells, and that microglia are participating in the clearance of PEDOT:PSS:EG from the implantation site. Horizontal brain sections at 1-month post-implantation also revealed microglial cells (in red) localizing in the region where PEDOT:PSS:EG (in green) is delaminated or directly in contact with the brain (Fig. 7 d). These cells are CD68 positive (in white), the presence of intracellular phagocytic structures. Based on the results discussed above, we hypothesise that implanted TNPs lead to the formation of a less tight glial scar compared to PI probes. As a consequence, microglia have the space needed to access the probe and phagocyte the delaminated PEDOT:PSS:EG flakes.

\subsection{Probe's degradation}

One of the most exciting features of transient medical devices is their natural ability to disappear in the body, which in turns reduces the longterm foreign body reaction. PCL bulk degradation occurs slowly with a time scale spanning several months or years [23], thus making an in-vivo assessment in animals extremely challenging. In order to investigate the degradation process of all-polymeric TNPs, we performed in-vitro accelerated degradation tests, by leaving 3 TNPs immersed in PBS at $37^{\circ} \mathrm{C}$ and $\mathrm{pH} 12$ and monitoring their weight over time. Scanning electron microscope images showed the appearance of micro- and macro-cracks over time due to hydrolysis (Fig. 8a), and all-polymeric 

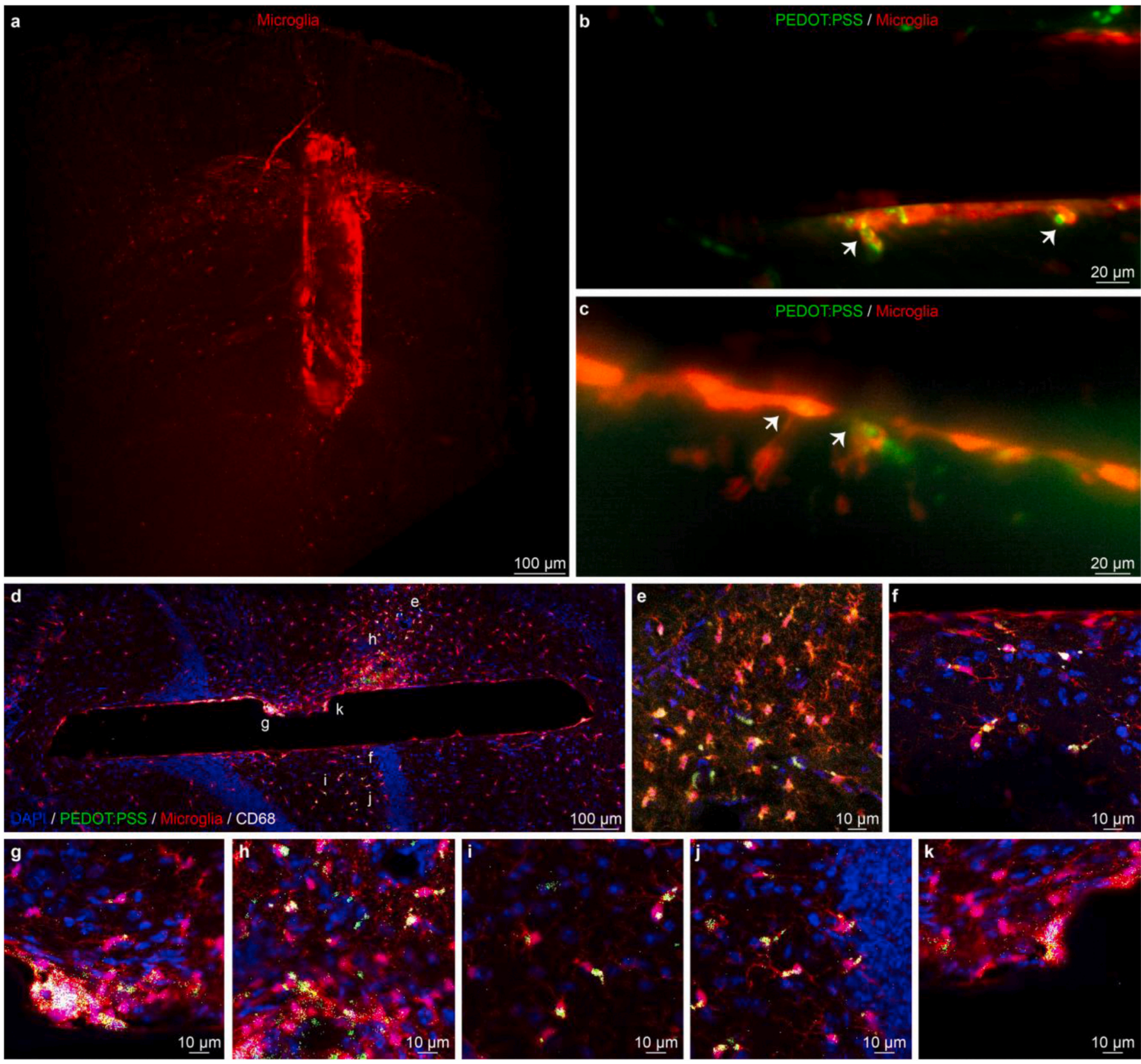

Fig. 7. | Integration with microglia. a, 3D mesoSPIM image of a clarified brain sample with fluorescent microglia (red) implanted with a TNP with FITC-labelled PEDOT:PSS at 1-month post-implantation. b,c, COLM images of the probe surface, showing fluorescent PEDOT:PSS (in green) and microglia (in red) colocalization 1-month (b) and 2-months (c) post-implantation. Microglia engulf PEDOT:PSS:EG particles which are detaching from the probe (white arrows). d, Horizontal brain section of mouse brain with fluorescent microglia (in red) implanted with a fluorescent TNP (without encapsulation) at 1-month post-implantation. e,k, Various magnifications of the section in d (corresponding letters) showing phagocytic microglia engulfing PEDOT:PSS:EG (in green). CD68 (in white) is a specific marker for microglial phagocytic structures. (For interpretation of the references to colour in this figure legend, the reader is referred to the Web version of this article.)

TNPs undergo full degradation in about one year of time at $\mathrm{pH} 12$ (Fig. 8b). A comparison between the days to reach a weight loss of $5 \%$ at $\mathrm{pH} 12$ and at $\mathrm{pH} 7.4$ revealed that the test has an acceleration factor of about 2.5. Slow degradation is an appealing feature for TNPs, since it might allow for better tissue remodelling and reduced chronic trauma at the implantation site $[26,52,53]$. To provide evidence of slow degradation in-vivo of all-polymeric TNPs, we implanted them in the cortex of mice and subsequently performed immunofluorescence assays on horizontal brain slices at a fixed time point. Upon long-term implantation (9 months), the all-polymeric TNPs showed a minor chronic glial scar (Fig. 8c), evaluated with staining for astrocytes against GFAP. The formation of a glial scar should be minimised or avoided for several reasons: it increases the distance between the recording surface of the electrode and neurons, eventually impairing the recording capabilities of the device $[54,55]$, and acts as a barrier for growth factors, ions, and signalling molecules, thus promoting neural apoptosis and impeding axon regeneration $[56,57]$. In the case of TNPs, the presence of a limited glial scar allows tissue remodelling around the probe, and, as a consequence, neurons (labelled against NeuN in green) are free to relocate within the device, which serves as a support scaffold during the degradation (Fig. 8d). This evidence of the probe slow degradation in-vivo is a promising sign of reduced chronic trauma.

\section{Discussion}

In biomedical engineering and medicine, transient devices are a 

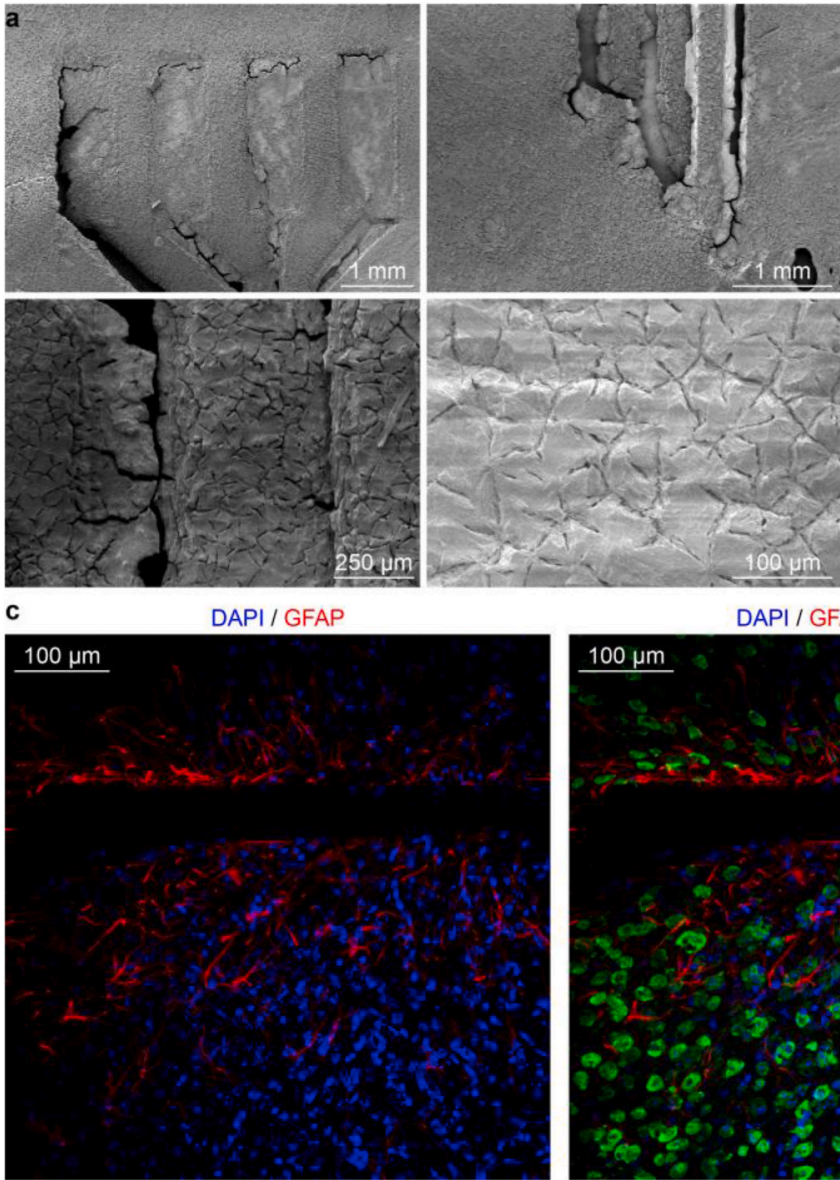

DAPI / GFAP / NeuN

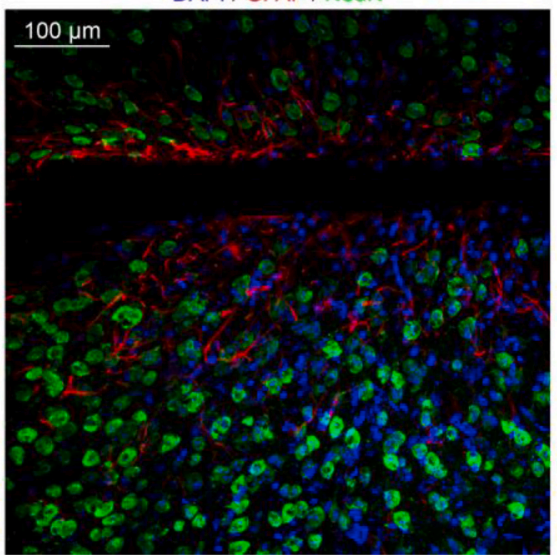

b

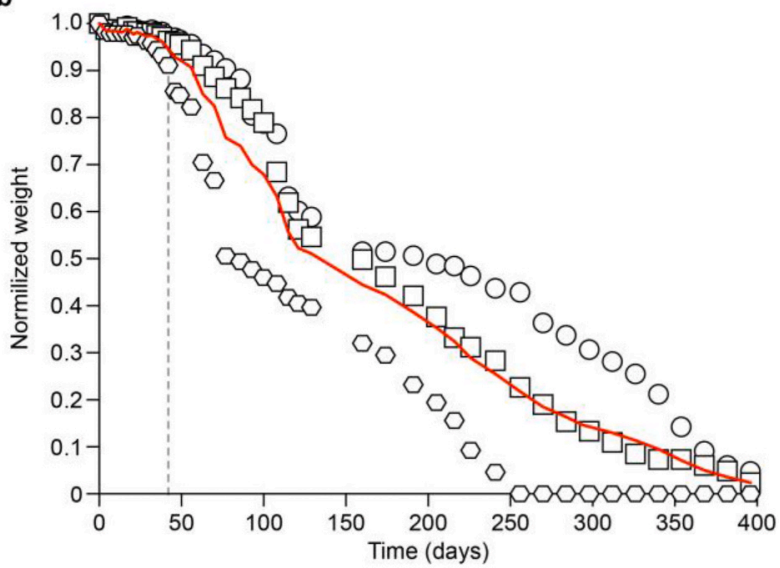

d

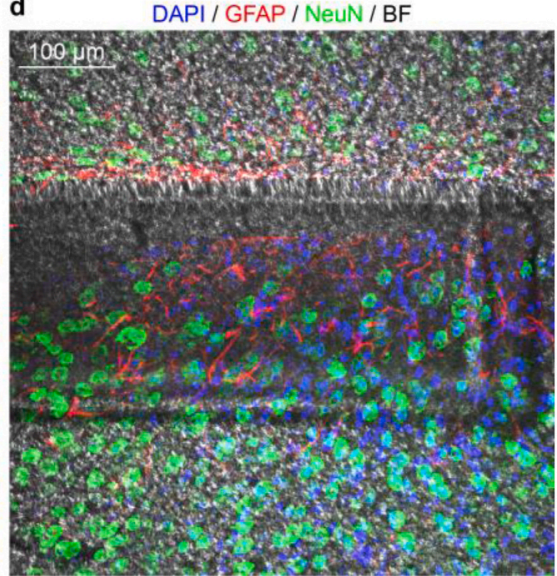

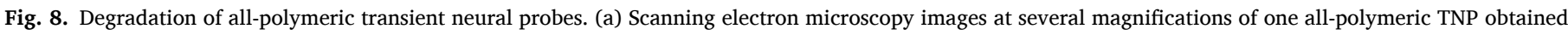

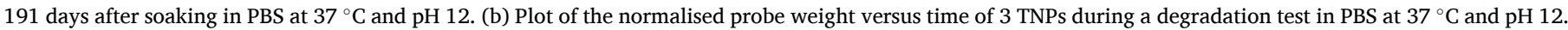

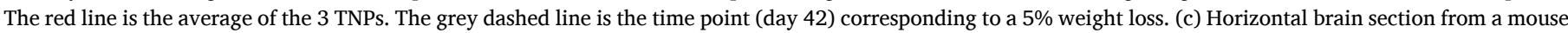

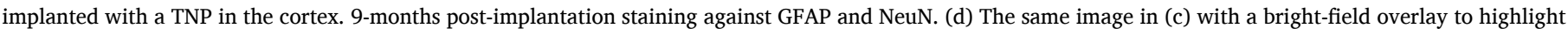
the area occupied by the TNP. (For interpretation of the references to colour in this figure legend, the reader is referred to the Web version of this article.)

relatively new concept which has the potential to widen the range of possible applications of implantable medical devices. We explored this new concept with using fully polymer-based devices to exploit their unique characteristics and try to overcome some of the current limitations. So far, transient medical devices showed a short functional lifetime because of the fast degradation of degradable metals in contact with body fluids $[11,18]$.

In this work, we showed the fabrication and characterization of a polymer-based implantable device conceived to disappear after being functional for a few months rather than a few days. A longer device lifetime will allow, for instance, the development of prosthetic devices for mid-term monitoring of the brain activity, either before or after surgery in chronic epileptic patients. Nowadays, clinicians make decisions on localised surgical treatments based on intracranial electroencephalography recordings that are typically lasting for only 1 or 2 weeks in favour of patient compliance. On the other hand, recent publications in this field revealed that intracranial electroencephalography recordings do not stabilize until several weeks after implantation, due to the invasiveness of the procedure and the inflammatory response of the tissue $[58,59]$. Therefore, an extended chronic monitoring period, together with the possibility to mitigate the inflammatory reaction, would enable a more accurate representation of the spatiotemporal variability of the seizures, translating into a better diagnosis for the patient. Furthermore, a transient device with extended recording capability would allow for continuous monitoring after the surgical procedure, thus providing essential insights into the brain activity during the recovery period and, possibly, into the effects of the following pharmaceutical treatment. Also, the absence of metals in the implanted portion of the device would provide magnetic resonance imaging compatibility $[29,60,61]$, allowing the pairing of the electrophysiological monitoring with structural and functional images, therefore obtaining a clearer picture of the brain activity during the recovery period.

Even though only a few electrodes (approximately 20\%) were still operational three months post-implantation, we believe that there is still room for fabrication strategies that can improve the device lifetime, such as enhancing the adhesion between PEDOT:PSS and PCL by using additives or by oxygen plasma treatment. Also, even if a performance comparison with other inorganic and not-transient devices is out of the scope of this study (due to the transient nature of the presented device), the electrochemical characterization of all-polymeric TNPs showed a stable performance within a wide range of frequencies, thus allowing us to perform acute and chronic in-vivo recordings in mice with excellent signal to noise ratio.

Recently, not-transient implantable neural probes reached extreme miniaturisation to reduce chronic foreign body reaction [62,63]. Miniaturisation for the TNP is limited by materials properties and fabrication methods. However, a detailed study of the foreign body reaction revealed that all-polymeric TNPs were responsible for a minimal glial scar around the implant. As a consequence, neurons were able to colonize the slowly degrading PCL layers, hence promoting tissue remodelling: such behaviour is the opposite to what is usually observed with 
permanent implants of similar sizes, where neurons are depleted from the implantation site [54].

In summary, the in-vivo validation and the degradation study, together with the preliminary evidence of phagocytic microglia engulfing the conductive polymer, demonstrate that the combination of the transient electronic approach and the usage of polymers is a new promising path to improve the device-tissue interface, extend the durability of implanted transient devices, and increase their possible applications. Although further research is needed to fully understand the fate of microglial cells upon phagocytosis of PEDOT:PSS:EG, these results can drive the conceptualization of new conductive polymers with links could be degradable, for instance, by resident microglia.

\section{Declaration of competing interest}

The authors declare that they have no known competing financial interests or personal relationships that could have appeared to influence the work reported in this paper.

\section{Acknowledgement}

This work has been supported by École polytechnique fédérale de Lausanne, Medtronic PLC and European Commission (Grant agreement 701632). The authors would like to thank Dr. Laura Batti and Audrey Tissot for the support with the brain clarification process and imaging and Vivien Gaillet for the drawings.

\section{Appendix A. Supplementary data}

Supplementary data to this article can be found online at https://doi. org/10.1016/j.biomaterials.2021.120889.

\section{Data availability}

The authors declare that all other relevant data supporting the findings of the study are available in this article and in its supplementary information file. Access to our raw data can be obtained from the corresponding author upon reasonable request.

\section{Credit author statement}

Laura Ferlauto: designed the study, fabricated the devices, performed electrochemical characterization and analysed data, performed surgeries, performed immunohistochemistry, collected confocal images, and wrote the manuscript. Paola Vagni: designed the study, performed surgeries, performed electrophysiology and analysed data, performed immunohistochemistry, collected and analysed confocal images, and wrote the manuscript. Adele Fanelli: fabricated the devices, performed electrochemical characterization, performed the accelerated degradation study in-vitro, and analysed data. Elodie Geneviève Zollinger: performed the surgeries and immunohistochemistry, collected confocal images, and performed cytotoxicity assay. Katia Monsorno and Rosa Chiara Paolicelli: supplied genetically modified mice and participated in the histological tests. Diego Ghezzi: supervised the entire study and wrote the manuscript. All the authors read and accepted the manuscript.

\section{References}

[1] T. Lei, M. Guan, J. Liu, H.-C.C. Lin, R. Pfattner, L. Shaw, A.F. McGuire, T.-C. C. Huang, L. Shao, K.-T.T. Cheng, J.B. Tok, Z. Bao, Biocompatible and totally disintegrable semiconducting polymer for ultrathin and ultralightweight transient electronics, in: Proceedings of the National Academy of Sciences of the United States of America, 2017, https://doi.org/10.1073/pnas.1701478114.

[2] G.A. Salvatore, J. Sülzle, F. Dalla Valle, G. Cantarella, F. Robotti, P. Jokic, S. Knobelspies, A. Daus, L. Büthe, L. Petti, N. Kirchgessner, R. Hopf, M. Magno, G. Tröster, Biodegradable and highly deformable temperature sensors for the internet of things, Adv. Funct. Mater. 27 (2017) 1702390, https://doi.org/ $10.1002 /$ adfm. 201702390.
[3] C. Dagdeviren, S.-W. Hwang, Y. Su, S. Kim, H. Cheng, O. Gur, R. Haney, F. G. Omenetto, Y. Huang, J.A. Rogers, Transient, biocompatible electronics and energy harvesters based on ZnO, Small 9 (2013) 3398-3404, https://doi.org/ 10.1002/smll.201300146.

[4] L. Yin, X. Huang, H. Xu, Y. Zhang, J. Lam, J. Cheng, J.A. Rogers, Materials, designs, and operational characteristics for fully biodegradable primary batteries, Adv. Mater. 26 (2014) 3879-3884, https://doi.org/10.1002/adma.201306304.

[5] G. Lee, S.-K. Kang, S.M. Won, P. Gutruf, Y.R. Jeong, J. Koo, S.-S. Lee, J.A. Rogers, J. S. Ha, Fully biodegradable microsupercapacitor for power storage in transient electronics, Adv. Energy Mater. 7 (2017) 1700157, https://doi.org/10.1002/ aenm. 201700157.

[6] S.-W.W. Hwang, H. Tao, D.-H.H. Kim, H. Cheng, J.-K.K. Song, E. Rill, M. A. Brenckle, B. Panilaitis, S.M. Won, Y.-S.S. Kim, Y.M. Song, K.J. Yu, A. Ameen, R. Li, Y. Su, M. Yang, D.L. Kaplan, M.R. Zakin, M.J. Slepian, Y. Huang, F. G. Omenetto, J.A. Rogers, A physically transient form of silicon electronics, Science (New York, N.Y.). 337 (2012) 1640-1644, https://doi.org/10.1126/ science.1226325.

[7] J. Koo, M.R. MacEwan, S.-K. Kang, S.M. Won, M. Stephen, P. Gamble, Z. Xie, Y. Yan, Y.-Y. Chen, J. Shin, N. Birenbaum, S. Chung, S.B. Kim, J. Khalifeh, D. V. Harburg, K. Bean, M. Paskett, J. Kim, Z.S. Zohny, S.M. Lee, R. Zhang, K. Luo, B. Ji, A. Banks, H.M. Lee, Y. Huang, W.Z. Ray, J.A. Rogers, Wireless bioresorbable electronic system enables sustained nonpharmacological neuroregenerative therapy, Nat. Med. 24 (2018) 1830-1836, https://doi.org/10.1038/s41591-0180196-2.

[8] C.M. Boutry, L. Beker, Y. Kaizawa, C. Vassos, H. Tran, A.C. Hinckley, R. Pfattner, S. Niu, J. Li, J. Claverie, Z. Wang, J. Chang, P.M. Fox, Z. Bao, Biodegradable and flexible arterial-pulse sensor for the wireless monitoring of blood flow, Nat. Biomed. Eng. 3 (2019) 47-57, https://doi.org/10.1038/s41551-018-0336-5.

[9] H. Tao, S.-W. Hwang, B. Marelli, B. An, J.E. Moreau, M. Yang, M.A. Brenckle, S. Kim, D.L. Kaplan, J.A. Rogers, F.G. Omenetto, Silk-based resorbable electronic devices for remotely controlled therapy and in vivo infection abatement, Proc. Natl. Acad. Sci. Unit. States Am. 111 (2014) 17385-17389, https://doi.org/ 10.1073/pnas.1407743111.

[10] L. Lu, Z. Yang, K. Meacham, C. Cvetkovic, E.A. Corbin, A. Vázquez-Guardado, M. Xue, L. Yin, J. Boroumand, G. Pakeltis, T. Sang, K.J. Yu, D. Chanda, R. Bashir, R. W. Gereau, X. Sheng, J.A. Rogers, Biodegradable monocrystalline silicon photovoltaic microcells as power supplies for transient biomedical implants, Adv. Energy Mater. 8 (2018) 1703035, https://doi.org/10.1002/aenm.201703035.

[11] S.-W. Hwang, C.H. Lee, H. Cheng, J.-W. Jeong, S.-K. Kang, J.-H. Kim, J. Shin, J. Yang, Z. Liu, G.A. Ameer, Y. Huang, J.A. Rogers, Biodegradable elastomers and silicon nanomembranes/nanoribbons for stretchable, transient electronics, and biosensors, Nano Lett. 15 (2015) 2801-2808, https://doi.org/10.1021/ nl503997m.

[12] S.-K. Kang, R.K. Murphy, S.-W. Hwang, S. Lee, D.V. Harburg, N.A. Krueger, J. Shin, P. Gamble, H. Cheng, S. Yu, Z. Liu, J.G. McCall, M. Stephen, H. Ying, J. Kim, G. Park, C.R. Webb, C. Lee, S. Chung, D. Wie, A.D. Gujar, B. Vemulapalli, A.H. Kim, K.-M. Lee, J. Cheng, Y. Huang, S. Lee, P.V. Braun, W.Z. Ray, J.A. Rogers, Bioresorbable silicon electronic sensors for the brain, Nature 530 (2016) 71 https://doi.org/10.1038/nature16492.

[13] K. Xu, S. Li, S. Dong, S. Zhang, G. Pan, G. Wang, L. Shi, W. Guo, C. Yu, J. Luo, Bioresorbable electrode array for electrophysiological and pressure signal recording in the brain, Adv. Healthcare Mater. (2019), https://doi.org/10.1002/ adhm.201801649, 1801649.

[14] Implants that vanish, Nat. Biomed. Eng. 3 (2019), https://doi.org/10.1038/ s41551-019-0449-5, 585-585.

[15] M.A. Brenckle, H. Cheng, S. Hwang, H. Tao, M. Paquette, D.L. Kaplan, J.A. Rogers, Y. Huang, F.G. Omenetto, Modulated degradation of transient electronic devices through multilayer silk fibroin pockets, ACS Appl. Mater. Interfaces 7 (2015) 19870-19875, https://doi.org/10.1021/acsami.5b06059.

[16] K. Yu, D. Kuzum, S.-W. Hwang, B. Kim, H. Juul, N. Kim, S. Won, K. Chiang, M. Trumpis, A.G. Richardson, H. Cheng, H. Fang, M. Thompson, H. Bink, D. Talos, K. Seo, H. Lee, S.-K. Kang, J.-H. Kim, J. Lee, Y. Huang, F.E. Jensen, M.A. Dichter, T. H. Lucas, J. Viventi, B. Litt, J.A. Rogers, Bioresorbable silicon electronics for transient spatiotemporal mapping of electrical activity from the cerebral cortex, Nat. Mater. 15 (2016) 782-791, https://doi.org/10.1038/nmat4624.

[17] S.-W.W. Hwang, J.-K.K. Song, X. Huang, H. Cheng, S.-K.K. Kang, B.H. Kim, J.-H. H. Kim, S. Yu, Y. Huang, J.A. Rogers, High-performance biodegradable/transient electronics on biodegradable polymers, Adv. Mater. 26 (2014) 3905-3911, https:// doi.org/10.1002/adma.201306050.

[18] R. Li, L. Wang, D. Kong, L. Yin, Recent progress on biodegradable materials and transient electronics, Bioactive Mater. 3 (2018) 322-333, https://doi.org/ 10.1016/j.bioactmat.2017.12.001.

[19] X. Strakosas, M. Sessolo, A. Hama, J. Rivnay, E. Stavrinidou, G.G. Malliaras, R. M. Owens, A facile biofunctionalisation route for solution processable conducting polymer devices, J. Mater. Chem. B. 2 (2014) 2537-2545, https://doi.org/ 10.1039/C3TB21491E.

[20] E. Lee, J. Choi, Y. Jo, J.Y. Kim, Y.J. Jang, H.M. Lee, S.Y. Kim, H.-J. Lee, K. Cho, N. Jung, E.M. Hur, S.J. Jeong, C. Moon, Y. Choe, I.J. Rhyu, H. Kim, W. Sun, ACTPRESTO: rapid and consistent tissue clearing and labeling method for 3-dimensional (3D) imaging, Sci. Rep. 6 (2016) 18631, https://doi.org/10.1038/ srep18631.

[21] L. Asboth, L. Friedli, J. Beauparlant, C. Martinez-Gonzalez, S. Anil, E. Rey, L. Baud, G. Pidpruzhnykova, M.A. Anderson, P. Shkorbatova, L. Batti, S. Pagès, J. Kreider, B.L. Schneider, Q. Barraud, G. Courtine, Cortico-reticulo-spinal circuit reorganization enables functional recovery after severe spinal cord contusion, Nat. Neurosci. 21 (2018) 576-588, https://doi.org/10.1038/s41593-018-0093-5. 
[22] F.F. Voigt, D. Kirschenbaum, E. Platonova, S. Pagès, R.A.A. Campbell, R. Kastli, M. Schaettin, L. Egolf, A. van der Bourg, P. Bethge, K. Haenraets, N. Frézel, T. Topilko, P. Perin, D. Hillier, S. Hildebrand, A. Schueth, A. Roebroeck, B. Roska, E.T. Stoeckli, R. Pizzala, N. Renier, H.U. Zeilhofer, T. Karayannis, U. Ziegler, L. Batti, A. Holtmaat, C. Lüscher, A. Aguzzi, F. Helmchen, The mesoSPIM initiative: open-source light-sheet microscopes for imaging cleared tissue, Nat. Methods 16 (2019) 1105-1108, https://doi.org/10.1038/s41592-019-0554-0.

[23] M.A. Woodruff, D.W. Hutmacher, The return of a forgotten polymer-polycaprolactone in the 21st century, Prog. Polym. Sci. 35 (2010) 1217-1256, https://doi.org/10.1016/j.progpolymsci.2010.04.002.

[24] C.E. Nyitray, R. Chang, G. Faleo, K.D. Lance, D.A. Bernards, Q. Tang, T.A. Desai, Polycaprolactone thin-film micro- and nanoporous cell-encapsulation devices, ACS Nano 9 (2015) 5675-5682, https://doi.org/10.1021/acsnano.5b00679.

[25] E. Schlesinger, N. Ciaccio, T.A. Desai, Polycaprolactone thin-film drug delivery systems: empirical and predictive models for device design, Mater. Sci. Eng. C 57 (2015) 232-239, https://doi.org/10.1016/j.msec.2015.07.027.

[26] U. Sharma, D. Concagh, L. Core, Y. Kuang, C. You, Q. Pham, G. Zugates, R. Busold, S. Webber, J. Merlo, R. Langer, G.M. Whitesides, M. Palasis, The development of bioresorbable composite polymeric implants with high mechanical strength, Nat. Mater. 17 (2017) 96, https://doi.org/10.1038/nmat5016.

[27] G. Cellot, P. Lagonegro, G. Tarabella, D. Scaini, F. Fabbri, S. Iannotta, M. Prato, G. Salviati, L. Ballerini, PEDOT:PSS interfaces support the development of neurona synaptic networks with reduced neuroglia response in vitro, Front. Neurosci. 9 (2016), https://doi.org/10.3389/fnins.2015.00521.

[28] D.A. Koutsouras, A. Hama, J. Pas, P. Gkoupidenis, B. Hivert, C. Faivre-Sarrailh, E. Di Pasquale, R.M. Owens, G.G. Malliaras, PEDOT:PSS microelectrode arrays for hippocampal cell culture electrophysiological recordings, MRC 7 (2017) 259-265, https://doi.org/10.1557/mrc.2017.34.

[29] E. Cuttaz, J. Goding, C. Vallejo-Giraldo, U. Aregueta-Robles, N. Lovell, D. Ghezzi, R.A. Green, Conductive elastomer composites for fully polymeric, flexible bioelectronics, Biomater. Sci. 7 (2019) 1372-1385, https://doi.org/10.1039/ C8BM01235K.

[30] Y. Liu, J. Liu, S. Chen, T. Lei, Y. Kim, S. Niu, H. Wang, X. Wang, A.M. Foudeh, J.B.H. Tok, Z. Bao, Soft and elastic hydrogel-based microelectronics for localized lowvoltage neuromodulation, Nat. Biomed. Eng. 3 (2019) 58-68, https://doi.org/ 10.1038/s41551-018-0335-6.

[31] L. Ferlauto, A.N. D'Angelo, P. Vagni, M.J.I.A. Leccardi, F.M. Mor, E.A. Cuttaz, M. O. Heuschkel, L. Stoppini, D. Ghezzi, Development and characterization of PEDOT: PSS/alginate soft microelectrodes for application in neuroprosthetics, Front. Neurosci. 12 (2018) 648, https://doi.org/10.3389/fnins.2018.00648.

[32] V.R. Feig, H. Tran, Z. Bao, Biodegradable Polymeric Materials in Degradable Electronic Devices, ACS Central Science, 2018, https://doi.org/10.1021/ acscentsci.7b00595.

[33] Y.-J. (林祐仲 ), W.-S. Lin, 倪維仕, J.-Y. Ni, 李哲佑, Lee, Effect of incorporation of ethylene glycol into PEDOT:PSS on electron phonon coupling and conductivity, J. Appl. Phys. 117 (2015) 215501, https://doi.org/10.1063/1.4921930.

[34] Q. Wei, M. Mukaida, Y. Naitoh, T. Ishida, Morphological change and mobility enhancement in PEDOT:PSS by adding Co-solvents, Adv. Mater. 25 (2013) 2831-2836, https://doi.org/10.1002/adma.201205158.

[35] A. Blau, A. Murr, S. Wolff, E. Sernagor, P. Medini, G. Iurilli, C. Ziegler, F. Benfenati, Flexible, all-polymer microelectrode arrays for the capture of cardiac and neuronal signals, Biomaterials 32 (2011) 1778-1786, https://doi.org/10.1016/j. biomaterials.2010.11.014.

[36] L.D. Garma, L.M. Ferrari, P. Scognamiglio, F. Greco, F. Santoro, Inkjet-printed PEDOT:PSS multi-electrode arrays for low-cost in vitro electrophysiology, Lab Chip 19 (2019) 3776-3786, https://doi.org/10.1039/C9LC00636B.

[37] Y. Liu, J. Li, S. Song, J. Kang, Y. Tsao, S. Chen, V. Mottini, K. McConnell, W. Xu, Y. Q. Zheng, J.B.-H. Tok, P.M. George, Z. Bao, Morphing electronics enable neuromodulation in growing tissue, Nat. Biotechnol. 38 (2020) 1031-1036, https://doi.org/10.1038/s41587-020-0495-2.

[38] A. Lüttjohann, P.F. Fabene, G. van Luijtelaar, A revised Racine's scale for PTZinduced seizures in rats, Physiol. Behav. 98 (2009) 579-586, https://doi.org/ 10.1016/j.physbeh.2009.09.005.

[39] N. Javadian, N. Rahimi, M. Javadi-Paydar, A.H. Doustimotlagh, A.R. Dehpour, The modulatory effect of nitric oxide in pro- and anti-convulsive effects of vasopressin in PTZ-induced seizures threshold in mice, Epilepsy Res. 126 (2016) 134-140, https://doi.org/10.1016/j.eplepsyres.2016.07.006.

[40] W.D. Yonekawa, H.J. Kupferberg, D.M. Woodbury, Relationship between pentylenetetrazol-induced seizures and brain pentylenetetrazol levels in mice, J. Pharmacol. Exp. Therapeut. 214 (1980) 589-593.

[41] A. Schander, H. Stemmann, E. Tolstosheeva, R. Roese, V. Biefeld, L. Kempen, A. K. Kreiter, W. Lang, Design and fabrication of novel multi-channel floating neural probes for intracortical chronic recording, Sensor Actuator Phys. 247 (2016) 125-135, https://doi.org/10.1016/j.sna.2016.05.034.

[42] H. Charkhkar, G.L. Knaack, D.G. McHail, H.S. Mandal, N. Peixoto, J.F. Rubinson, T. C. Dumas, J.J. Pancrazio, Chronic intracortical neural recordings using microelectrode arrays coated with PEDOT-TFB, Acta Biomater. 32 (2016) 57-67, https://doi.org/10.1016/j.actbio.2015.12.022.

[43] K.A. Ludwig, J.D. Uram, J. Yang, D.C. Martin, D.R. Kipke, Chronic neural recordings using silicon microelectrode arrays electrochemically deposited with a poly(3,4-ethylenedioxythiophene) (PEDOT) film, J. Neural. Eng. 3 (2006) 59-70, https://doi.org/10.1088/1741-2560/3/1/007.

[44] A. Schander, S. Strokov, H. Stemmann, T. Tebmann, A.K. Kreiter, W. Lang, A flexible 202-channel epidural ECoG array with PEDOT: PSS coated electrodes for chronic recording of the visual cortex, IEEE Sensor. J. 19 (2019) 820-825, https:// doi.org/10.1109/JSEN.2018.2880833.

[45] A. Fanelli, L. Ferlauto, E.G. Zollinger, O. Brina, P. Reymond, P. Machi, D. Ghezzi, Degradable endovascular neural interface for minimally invasive neural recording and stimulation, Bioengineering (2021), https://doi.org/10.1101/ 2021.03.24.436737.

[46] F. Gong, C. Meng, J. He, X. Dong, Fabrication of highly conductive and multifunctional polyester fabrics by spray-coating with PEDOT:PSS solutions, Prog. Org. Coating 121 (2018) 89-96, https://doi.org/10.1016/j.porgcoat.2018.04.006.

[47] V. Sethumadhavan, K. Zuber, C. Bassell, P.R. Teasdale, D. Evans, Hydrolysis of doped conducting polymers, Commun. Chem. 3 (2020) 153, https://doi.org/ 10.1038/s42004-020-00404-y.

[48] E.M. Thaning, M.L.M. Asplund, T.A. Nyberg, O.W. Inganäs, H. von Holst, Stability of poly(3,4-ethylene dioxythiophene) materials intended for implants, J. Biomed. Mater. Res. 93B (2010) 407-415, https://doi.org/10.1002/jbm.b.31597.

[49] T. Chen, Y. Lin, X. Bi, Y. Gu, Conductive poly(3,4-ethylenedioxythiophene) is effectively degradable by hydrogen peroxide with iron (II) chloride, Mater. Chem. Phys. 242 (2020) 122509, https://doi.org/10.1016/j.matchemphys.2019.122509.

[50] H. Gong, J. Xiang, L. Xu, X. Song, Z. Dong, R. Peng, Z. Liu, Stimulation of immune systems by conjugated polymers and their potential as an alternative vaccine adjuvant, Nanoscale 7 (2015) 19282-19292, https://doi.org/10.1039/ C5NR06081H.

[51] R. Fu, Q. Shen, P. Xu, J.J. Luo, Y. Tang, Phagocytosis of microglia in the central nervous system diseases, Mol. Neurobiol. 49 (2014) 1422-1434, https://doi.org/ 10.1007/s12035-013-8620-6.

[52] J. Shin, Y. Yan, W. Bai, Y. Xue, P. Gamble, L. Tian, I. Kandela, C.R. Haney, W. Spees, Y. Lee, M. Choi, J. Ko, H. Ryu, J.-K. Chang, M. Pezhouh, S.-K. Kang, S. M. Won, K.J. Yu, J. Zhao, Y.K. Lee, M.R. MacEwan, S.-K. Song, Y. Huang, W.Z. Ray, J.A. Rogers, Bioresorbable pressure sensors protected with thermally grown silicon dioxide for the monitoring of chronic diseases and healing processes, Nat. Biomed. Eng. 3 (2019) 37-46, https://doi.org/10.1038/s41551-018-0300-4.

[53] Y. Su, I. Cockerill, Y. Wang, Y.-X. Qin, L. Chang, Y. Zheng, D. Zhu, Zinc-based biomaterials for regeneration and therapy, Trends Biotechnol. 37 (2019) 428-441, https://doi.org/10.1016/j.tibtech.2018.10.009.

[54] M. Jorfi, J.L. Skousen, C. Weder, J.R. Capadona, Progress towards biocompatible intracortical microelectrodes for neural interfacing applications, J. Neural. Eng. 12 (2015), 011001, https://doi.org/10.1088/1741-2560/12/1/011001.

[55] D. Scaini, L. Ballerini, Nanomaterials at the neural interface, Curr. Opin. Neurobiol. 50 (2018) 50-55, https://doi.org/10.1016/j.conb.2017.12.009.

[56] S.M. Wellman, T.D.Y. Kozai, Understanding the inflammatory tissue reaction to brain implants to improve neurochemical sensing performance, ACS Chem. Neurosci. 8 (2017) 2578-2582, https://doi.org/10.1021/acschemneuro.7b00403.

[57] J.M. Cregg, M.A. DePaul, A.R. Filous, B.T. Lang, A. Tran, J. Silver, Functional regeneration beyond the glial scar, Exp. Neurol. 253 (2014) 197-207, https://doi. org/10.1016/j.expneurol.2013.12.024.

[58] H. Ung, S.N. Baldassano, H. Bink, A.M. Krieger, S. Williams, F. Vitale, C. Wu, D. Freestone, E. Nurse, K. Leyde, K.A. Davis, M. Cook, B. Litt, Intracranial EEG fluctuates over months after implanting electrodes in human brain, J. Neural. Eng. 14 (2017), 056011, https://doi.org/10.1088/1741-2552/aa7f40.

[59] H. Ung, K.A. Davis, D. Wulsin, J. Wagenaar, E. Fox, J.J. McDonnell, N. Patterson, C. H. Vite, G. Worrell, B. Litt, Temporal behavior of seizures and interictal bursts in prolonged intracranial recordings from epileptic canines, Epilepsia 57 (2016) 1949-1957, https://doi.org/10.1111/epi.13591.

[60] P. Li, D. Du, L. Guo, Y. Guo, J. Ouyang, Stretchable and conductive polymer films for high-performance electromagnetic interference shielding, J. Mater. Chem. C. 4 (2016) 6525-6532, https://doi.org/10.1039/C6TC01619G.

[61] Y. Wang, X. Jing, Intrinsically conducting polymers for electromagnetic interference shielding, Polym. Adv. Technol. 16 (2005) 344-351, https://doi.org/ 10.1002/pat.589.

[62] L. Luan, X. Wei, Z. Zhao, J.J. Siegel, O. Potnis, C.A. Tuppen, S. Lin, S. Kazmi, R. A. Fowler, S. Holloway, A.K. Dunn, R.A. Chitwood, C. Xie, Ultraflexible nanoelectronic probes form reliable, glial scar-free neural integration, Sci. Adv. 3 (2017), e1601966, https://doi.org/10.1126/sciadv.1601966.

[63] X. Yang, T. Zhou, T.J. Zwang, G. Hong, Y. Zhao, R.D. Viveros, T.-M. Fu, T. Gao, C. M. Lieber, Bioinspired neuron-like electronics, Nat. Mater. 18 (2019) 510-517, https://doi.org/10.1038/s41563-019-0292-9. 